\title{
Tim-3 drives tissue Treg effector function to promote neonatal immune tolerance and tumor- induced immune suppression
}

\section{Min Yang}

University of Pittsburgh

Shaoxian Wu

University of Pittsburgh

Wenyan Zhou

University of Pittsburgh

Chunyan He

University of Pittsburgh

\section{Runzi Sun}

University of Pittsburgh

Yao-yu Ying

Medical College of Soochow University

\section{Yi Zhou}

University of Pittsburgh

\section{Sihan Li}

University of Pittsburgh

Wensi Zhai

University of Pittsburgh

Bendong Chen

University of Pittsburgh

Jun Xue

University of Pittsburgh

\section{Da Yang}

Department of Pharmaceutical Sciences, University of Pittsburgh https://orcid.org/0000-0002-83369457

Binfeng Lu ( $\sim$ binfeng@pitt.edu )

University of Pittsburgh https://orcid.org/0000-0002-7822-5950

Article 
Keywords: Tissue regulatory T cells, immune suppressive tumor microenvironment, neonatal immune tolerance, tumor-induced immune suppression

Posted Date: July 17th, 2020

DOI: https://doi.org/10.21203/rs.3.rs-38445/v1

License: (c) (1) This work is licensed under a Creative Commons Attribution 4.0 International License. Read Full License 
Tim-3 drives tissue Treg effector function to promote neonatal immune tolerance and tumor-induced immune suppression

Min Yang ${ }^{1}$, Shaoxian $\mathrm{Wu}^{1}$, Wenyan Zhou ${ }^{1}$, Chunyan $\mathrm{He}^{1}$, Runzi Sun ${ }^{1}$, Yaoyu Ying ${ }^{1}$, Yi Zhou ${ }^{1}$, Sihan $\mathrm{Li}^{2}$, Wensi Zhai ${ }^{1}$, Bendong Chen ${ }^{1}$, Jun Xue ${ }^{1}$, Da Yang ${ }^{2}$, Binfeng Lu ${ }^{1 *}$

${ }^{1}$ Department of Immunology, University of Pittsburgh, PA, 15261, USA

${ }^{2}$ Department of Pharmaceutical Sciences, University of Pittsburgh, PA 15261, USA

*Correspondence: $\underline{\text { binfeng@pitt.edu }}$

\section{Abstract:}

Tissue regulatory T cells (Treg) are a key player in preventing immune pathology and shaping the immune suppressive tumor microenvironment (TME). The signaling molecules that uniquely regulate tissue Treg are not well understood. Here, we show that Tim-3 was predominantly expressed in Treg cells in both normal and tumor tissues. Notably, Tim-3 deficiency in Tregs led to the development of severe signs of autoimmune diseases in neonatal mice, indicating a crucial role of Tim-3 in establishing immune tolerance in early life. In addition, deletion of Tim-3 on Tregs resulted in reduced numbers and suppressor activities of tumoral Tregs and an increase in antitumor immune responses. A gene profiling study revealed the requirement of Tim-3 in upregulation of effector Treg genes encoding immune inhibitory molecules, chemokine receptors for tissue residency, chemokines, and 
cytokines. Moreover, Tim-3 deficiency in Tregs and treatment with PD-1 mAbs synergistically inhibited tumor growth. Our study demonstrates the essential role of Tim-3 in driving tissue effector Treg-mediated immune suppression. 


\section{Introduction:}

Regulatory $\mathrm{T}$ (Treg) cells play a key part in preventing autoimmunity and maintaining immune tolerance in cancer and transplantation (1). Treg cells that are present in lymphoid tissues have been extensively investigated. Comparatively, Treg cells that reside in the non-lymphoid tissues, called tissue Tregs, are not well studied. Recent studies demonstrate that tissue Tregs, in small numbers, are found in many nonlymphoid healthy tissues, infected tissues, grafts, placenta, injured muscle, and tumors(2). It appears that tissue Tregs, similar to tissue conventional T cells, express genes that are upregulated in conventional effector, memory, and exhausted T cells. They also express chemokine receptors and adhesion molecules unique to various tissues. Moreover, tissue Treg cells have a distinct T cell receptor (TCR) repertoire indicating a local clonal expansion of these cells $(3,4)$. It is believed that these unique properties empower tissue Tregs to drive immune suppression in the tumor microenvironment (TME) (2). However, the specific molecular mechanisms that mediate the function of tissue Treg are not well understood.

Tim-3 was originally found upregulated in a Th1 cell line (5). Its expression was shown to be upregulated in chronically activated T cells upon multiple rounds of stimulation (6-8). It is upregulated and considered a marker for terminally differentiated T cells such as exhausted T cells (9-13). Besides conventional T cells, Tim-3 has recently been shown to be characteristically expressed in tissue Treg (2, 
14-18). Tim-3 was first reported to be specifically upregulated in tumor infiltrating regulatory T cells and its expression has been associated with tumor progression in human lung cancer(14). Tim-3 was also expressed in tissue resident Treg in normal tissues such as fat tissues (2). Recent studies have demonstrated that Tim $-3^{+}$Treg have effector like phenotype and greater suppressive activities than Tim-3- Treg (1519). In addition, combination treatement of tumor-bearing mice with PD-1 mAbs and Tim-3 mAbs led to a decrease in Treg function along with increased effector T cell function (17). The exact function of Tim-3 on tissue Tregs, however, remains unresolved.

In this study, we are aiming to shed light on the functional role of Tim-3 on Tregs. To this end, we generated Foxp3 specific Tim-3 deficient mice. Using this model, we examined the role of Tim-3 in Treg-mediated prevention of autoimmunity and tumor immune suppression. We also determined the function of Tim-3 on Tregs in tumor tolerance and tumor checkpoint-blockade immunotherapy. Furthermore, we performed whole transcriptome analysis to comprehensively study the molecular mechanism underlying Tim-3's function in tissue Treg cells. Our study establishes the functional role of Tim-3 in tissue Treg cells and the significance for tumor immunotherapy targeting Tim $-3^{+}$Treg cells.

\section{Results:}




\section{Mice with Tim-3 deficiency in Tregs developed severe signs of autoimmune}

\section{diseases}

We and others have previously demonstrated that Tim-3 is uniquely expressed on tissue regulatory T cells $(2,14)$. Here, we also showed that Tim- 3 was specifically up-regulated on skin tissue Treg cells from neonatal mice but was expressed at a minimal level in peripheral Treg cells (Fig1A). In order to elucidate the functional role of Tim-3 in Tregs, we generated Tim-3 flox/fox/Foxp3 $3^{\text {YFP-Cre }}$ (hereafter referred to as $T^{\text {f/f }}$ $F^{\text {Cre }}$ ) mice to specifically delete Tim-3 in Treg cells. Interestingly, $T^{f / f} F^{\text {Cre }}$ neonates quickly became runted around 14 days after birth and did not survive beyond 35 days (Fig. 1B). $T^{f / f} F^{C r e}$ mice also had scaly skins and tails as well as deformed ears, similar to the phenotype of scurfy mice (Fig. S1A) (20). Histological analysis showed hyper inflammation and mononuclear cell infiltration in skins and lungs from 18-day-old neonates (Fig. 1C and D and Fig. S1C). It was noted that lymph nodes were greatly enlarged in all $T^{\text {f/f }} F^{\text {Cre }}$ mice around 18 days after birth (Fig. S1A). Flow cytometry analysis demonstrated much lower percentages of Tregs in the skin and spleen but not lymph node in $T^{\text {f/f }} F^{\text {Cre }}$ mice compared to control wild type Tim-3 /Foxp3 $3^{\text {YFP-Cre }}$ mice $\left(T^{+/+} F^{C r e}\right)$ (Fig. 1E and F).

The analysis also showed that $T^{f / f} F^{C r e}$ mice had a higher percentage of both total immune cells and T cells in the skin when compared to wild type control mice (Fig1. $\mathrm{G}$ and $\mathrm{H}$ ). In addition, the percentage of conventional $\mathrm{CD} 4^{+}$and $\mathrm{CD} 8^{+} \mathrm{T}$ cells were 
also increased in the spleen of $T^{f / f} F^{C r e}$ mice compared to the percentage of those in control mice (Fig. S1D and E). Consistent with tissue inflammation and lymphadenopathy, higher percentages of $\mathrm{CD} 44^{+}$and $\mathrm{Ki} 67^{+}$conventional $\mathrm{T}$ cells and lower percentages of $\mathrm{CD} 62 \mathrm{~L}^{+}$conventional T cells were present in the skin, spleen, and lymph nodes of $\mathrm{T}^{\mathrm{f} / \mathrm{f}} \mathrm{F}^{\mathrm{Cre}}$ mice compared to control mice, indicating dysregulated activation of $\mathrm{T}$ lymphocytes in $\mathrm{T}^{\mathrm{f} / \mathrm{f}} \mathrm{F}^{\mathrm{Cre}}$ mice (Fig1. G, H, and Fig. S1D-G). Despite a huge increase of absolute number of cells in lymph node (FigS1B) and hyper activation of conventional T cells(Fig. S1F and G), the percentage of $\mathrm{CD}^{+} \mathrm{T}$ cells was decreased in lymph node of $T^{f / f} F^{C r e}$ mice. Collectively, these data suggest that Tim-3 expression on Tregs is crucial for maintaining immune tolerance homeostasis in neonates.

\section{Tim-3 deletion in Tregs resulted in a reduction in the number of tissue Tregs}

Although $T^{f / f} F^{C r e}$ mice developed severe signs of autoimmune diseases around 18 days after birth, the neonates still looked normal around 10 days. It is understood that Treg cells start to accumulate in skin beginning around postnatal day 6 and peak around day 13 (21). We then examined peripheral and skin immune cells in $T^{+/+} F^{\text {cre }}$ and $T^{T / f} F^{C r e}$ mice on postnatal day 10 by flow cytometry. The percentages of Tregs in spleens and lymph nodes were similar between $T^{+/+} F^{c r e}$ and $T^{f / f} F^{C r e}$ mice (Fig. S2A). In contrast, the percentages of Tregs in skin were significantly reduced in $T^{f / f} F^{\text {Cre }}$ mice compared to control mice (Fig. 2A and B). In addition, Tim-3 was not 
significantly expressed on skin Treg cells from $T^{\mathrm{f} / f} \mathrm{~F}^{\mathrm{Cre}}$ mice but expressed at high levels on those from control mice (Fig. 2A and B). In contrast to a large increase in CD $45^{+}$immune cells in the skin of 18 -day-old $T^{f / f} F^{C r e}$ mice, there were significantly less CD45 immune cells in the skin of 10 -day-old $T^{\text {fff }} F^{\text {Cre }}$ mice compared with control mice (Fig. 2A and B). However, both conventional $\mathrm{CD} 4^{+}$and $\mathrm{CD} 8^{+}$skin $\mathrm{T}$ cells expressed higher levels of activation markers such as CD44 and lower levels of naïve/ central memory T cells markers such as CD62L and IL7R (Fig. 2A and B) in $T^{f / f} F^{C r e}$ mice compared to control mice. In addition, conventional $\mathrm{CD} 4^{+}$and $\mathrm{CD} 8^{+} \mathrm{T}$ cells expressed higher levels of Ki67 in $T^{f / f} F^{C r e}$ mice compared to control mice (Fig. $2 A$ and $B$ ), suggesting that these cells were highly proliferative. Consistent with these changes in the tissue conventional T cells, the percentage of effector/memory $\mathrm{T}$ cells was also increased in the spleen (Fig. S2C and D) and slightly increased in lymph nodes (Fig. S2B) from $T^{f / f} F^{C r e}$ mice compared to control mice. No difference of thymic T cells was observed at this stage (data not shown). These data suggest that Tim-3 is required for the accumulation of tissue Tregs, and thus prevents uncontrolled activation and proliferation of $\mathrm{T}$ cells to maintain immune homeostasis at the early stage of life.

\section{Ablation of Tim-3 in Treg cells increased the immune surveillance of tumor}

Although $T^{f / f} F^{\text {Cre }}$ mice did not survive more than 35 days, approximately $40 \%$ of $\mathrm{Tim}$ $3^{\text {flox/ko }}$ Foxp $3^{\text {YFP-Cre }}$ (hereafter referred to as $T^{\text {T/- }} F^{\text {Cre }}$ ) mice, which had deleted one allele 
of Tim-3 in all cells and had one floxed Tim-3 allele, survived to adulthood (Fig. 3A). The lack of Tim-3 expression on some non-Treg immune cells in $T^{f /-} F^{C r e}$ mice likely dampened the early lethality phenotype of $T^{\text {f/f }} F^{C r e}$ mice. We then tried to determine the role of Tim-3 on Tregs in tumor immune surveillance using adult $T^{T / F^{C r e}}$ and control $T^{+/} F^{\text {Cre }}$ mice. We employed the MC-38 mouse colon cancer transplant tumor model. MC-38 cells formed tumors in $T^{+/} F^{\text {Cre }}$ mice and sustained tumor growth as in WT mice (Fig. 3B and data not shown). In contrast, the tumor growth was initially similar but eventually inhibited in $T^{f /-} F^{C r e}$ mice (Fig. 3B). We also generated Tim$3^{\text {floxflox }}$ Foxp $3^{\text {CreErt2GFP }}$ (called thereafter Tim- $3^{f / f} F^{\text {CreErt2 }}$ ) mice where we can inducibly

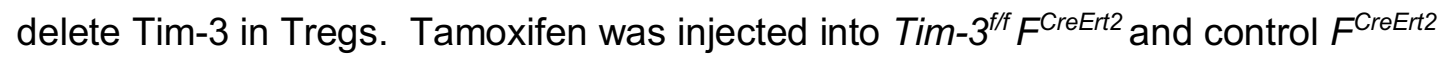
mice to induce deletion of Tim-3 in Tregs (Fig. S3A). We further found, inducible Tim3 deletion resulted in slower tumor growth (Fig.3C). These data suggest that Tim-3 expression on Tregs mediates tumor immune tolerance.

Analysis of tumor immune infiltrates was performed when tumor sizes were $7 \sim 8 \mathrm{~mm}$ and similar $T^{f /-} F^{C r e}$ and $T^{+/-} F^{C r e}$ mice. The data indicated that both the percentage and total number of $\mathrm{CD} 45^{+}$lymphocytes in $T^{f /} F^{C r e}$ mice were significantly greater than those in control group. In addition, percentages of both $\mathrm{CD} 4^{+}$and $\mathrm{CD} 8^{+} \mathrm{TIL}$ cells were increased in $T^{f / /} F^{C r e}$ mice when compared to control mice. (Fig. 3D and E). Consistent with our findings in skin inflammation, percentages of tumor infiltrating Tregs were reduced in $T^{f /} F^{\text {Cre }}$ mice (Fig.3D and E). Notably, Tim-3 was completely absent on Treg cells but expressed on conventional $\mathrm{CD} 4^{+}$and $\mathrm{CD} 8^{+} \mathrm{TIL}$ in $\mathrm{T}^{\mathrm{f} /-} \mathrm{F}^{\mathrm{Cre}}$ 
mice (Fig. 3D). In line with the reduction of Tregs cells, the effector molecules IFN- $\gamma$ in $\mathrm{CD}^{+} \mathrm{T}$ cells and granzyme $\mathrm{B}$ in $\mathrm{CD} 8^{+} \mathrm{T}$ cells were greatly increased in $T^{\mathrm{f} /} F^{\mathrm{Cre}}$ mice (Fig. 3D and E). In addition, the percentage of "exhausted" PD- $1^{+} \mathrm{Tim}-3^{+} \mathrm{CD} 8^{+} \mathrm{T}$ cells was reduced in $T^{f / /} F^{\text {Cre }}$ mice. Consistent to flow cytometry data, the IFN- $\gamma$ ELISpot assay demonstrated that greater numbers of tumor antigen-specific T cells were generated in $T^{T /} F^{C r e}$ mice, indicating enhanced tumor antigen-specific T cell immune responses (Fig. 3F and Fig. S3B). Analysis of the immune cell characteristics at a later stage when tumor sizes reached about $18 \mathrm{~mm}$ in diameter in control mice yielded similar results (Fig. S3C-D).

We also examined B16 melanoma, a poorly immunogenic tumor model, to further characterize the function of Tim-3 in tumoral Tregs. Tumor growth was similar between $T^{f / /} F^{C r e}$ and control mice (Fig. S3E). However, more CD45 $5^{+}$lymphocytes and $T$ cell infiltration were found in $T^{f / /} F^{C r e}$ mice than control mice (Fig. S3F-G). Again, the percentage of Foxp3 $^{+}$Treg cells was greatly reduced in $T^{f /-} F^{C r e}$ compared to control mice (Fig. S3F-G). Effector molecules of CD4 ${ }^{+}$TIL cells were also increased in $T^{f /-} F^{C r e}$ mice (Fig. S3F-G). In addition, CD44 and Ki67 expression of $\mathrm{CD} 4^{+}$TIL were increased, indicating enhanced activation and proliferation of CD4 ${ }^{+}$ TIL in $T^{f /-} F^{\text {Cre }}$ mice (Fig. S3F-G). These data suggest that Tim-3 deficiency in Treg cells leads to reduced immune suppression in the TME and a profound increase in anti-tumor immune responses. 


\section{Tim-3 is required for the suppressor function of tumor infiltrating Tregs.}

Tim $-3^{+}$tissue Treg cells have been shown to be more activated and suppressive in human cancer $(15-17,19)$. Given our data that mice with Tim-3 deficiency in Tregs leads to hyper activation and proliferation of conventional T cells and exhibited much greater antitumor immune responses, we sought to determine whether the suppression function of tumoral Treg cells is dependent on Tim-3. The suppression assay demonstrated that Tim-3 deficiency in Tregs resulted in a reduction of suppression activity (Fig. 4A and Fig. S4A). Flow cytometry analysis revealed that $T^{\text {t/- }}$ $F^{\text {Cre }}$ Treg cells expressed lower levels of CD44 and PD-1 but higher levels of IL7R, indicating that Tim-3 was required for the generation of effector Treg cells in the TME (Fig. 4B-C and Fig. S4B). Accordingly, Tim $-3^{+}$foxp3 $3^{+}$TIL from WT mice also expressed higher levels of CD44 and PD-1 and lower levels of IL7R (Fig. 4D-E). Consistent with the results from Tim-3 deficient mice, Tim-3 mAb treatment also resulted in a reduction of the percentage of tumoral Tregs. In addition, administration of Tim-3 mAb led to a decrease in CD44 and PD-1 expression and to an increase in IL7R expression in Tregs in the TME (Fig. 4F-G). These data suggest that Tim-3 is involved in the activation of Treg cells and also mediate immune suppressive functions of effector Tregs in tumor tissues. 
Tim-3 is required for driving a network of genes responsible for the function of effector Tregs.

To gain a comprehensive understanding of the role of Tim-3 in Tregs in the TME, we performed RNA-sequencing analysis comparing Tim-3-/- Tregs and control Tregs in the TME. There were more than 700 genes that were differentially expressed between $T^{f /-} F^{C r e}$ and control tumoral Tregs (Fig. 5A). Consistent with cytometric analysis, we found that Tim-3 deficient Treg cells expressed lower levels of CD44 but higher levels of IL7R and Tcf7, indicating Tim-3 is required for the effector phenotype of Treg cells (Fig. 5A, C). Gene enrichment analysis showed that control tumoral Tregs upregulated genes that were enriched with signature genes of Tim- $3^{+}$Tregs and genes highly expressed in Tim-3-/- Tregs were enriched with Tim-3- Treg signature genes (15) (Fig. 5B). These data indicated that Tim-3 signaling was required for the generation and function of Tim- $3^{+}$Treg cells in the TME. Gene enrichment analysis also indicated that genes upregulated in Tim-3 deficient tumoral Treg cells were enriched with signature genes of naïve $\mathrm{CD}^{+} \mathrm{T}$ cells and naïve Treg cells (Fig. 5B), suggesting that Tim-3 is required for the expression of effector Treg genes. Gene Ontology analysis revealed several categories of genes, such as cell cycle/apoptosis/survival, chemotaxis/adhesion, helper T cell subset differentiation, immune suppression and signaling, were increased in the Tim-3 sufficient tumoral Tregs, whereas several categories of genes including antigen presentation, cell cycle/apoptosis/survival, chemotaxis and adhesion, cytokine, differentiation, 
metabolism, signaling and stress response were enriched in Tim-3 deficient tumoral Treg cells (Fig. S5). Therefore, these data suggested that Tim-3 signaling is involved in regulating multitudes of cellular processes.

Further study of immunological function of the differentially expressed genes yielded an in depth understanding of how Tim-3 drove Treg-mediated immune suppression (Fig. 5C). We first focused on genes that were expressed at higher levels in WT Tregs than Tim-3 deficient Tregs. The analysis indicated that Tim-3 was required for the expression of chemokine receptor and adhesion molecules, such as Cxcr6, Itgb8, Itgav, Cx3cr1, Ccr8, and Cd44, which were likely involved in tissue residence of the effector/memory Treg cells (22-25). The reduced expression of these genes might explain the diminished number of Tim-3 deficient Treg cells in tumor tissues. It has been shown that the suppression function of Treg cells is dependent on their abilities to bring their target cells to proximity. This is controlled by chemokines such as $C c / 3, C c / 4, C c / 1, X c / 1$, and $C c / 12(15,26,27)$. Tim-3 was required for the upregulation of these chemokines in tumoral Tregs (Figure 5C). The analysis indicated that Tim-3 was critical for the expression of an array of immune suppressive effector genes such as Calca, Fcrl6, Stab1, F2rl2, I/10, Ramp1, Cd200, Lag3, Lgals4, Fasl, Entpd1 (28-38). Interestingly, avß8 integrin mRNAs were also expressed at a higher level in Tim-3 sufficient Tregs, suggesting Tim-3 expression confers Treg cells the ability to activate TGF- $\beta$ (39-41). Genes that were expressed higher in Tim-3 deficient Treg cells were mainly involved in function of the naïve and 
central/stem memory Treg cells, which was consistent with the idea that Tim-3 is involved in mediating the activation and function of effector Treg cells. Among these genes, several transcription factors, such as IL7R, KIf3, Tcf7, Twist2, Id3, Tsc22d3, Smad1, and KIf2, have been shown to be highly expressed in naïve and memory stem T cells (42). Higher expression of apoptotic and cell cycle inhibitor genes, such as $B c / 2 / 15, B c / 2, C d k n 2 b, C d k n 1 c$, and $C d k n 2 a$, were also consistent with the resting phenotype (42).

\section{Treg-specific deletion of Tim-3 gene synergized with PD-1 mAbs for tumor}

\section{therapy.}

It is thought that Tim-3 and PD-1 co-expression is the marker for exhausted CD8 ${ }^{+} \mathrm{T}$ cells and synergistic antitumor activities between PD-1 and Tim-3 mAbs are due to reversal of CD8 T cell exhaustion $(12,13)$. Since we have shown that both Tim-3 mAbs treatment and Tim-3 gene deletion in Tregs resulted in reduced percentage and activation of tumor infiltrating Tregs (Fig. 3C-D and Fig. 4B-C,F-G), we decided to determine whether PD-1 mAbs was synergistic with lack of Tim-3 in Tregs in producing antitumor efficacy. To this end, we treated MC38-bearing $T^{f /-} F^{C r e}$ and $T^{+/-}$ $F^{\text {Cre }}$ mice with control lgG or PD-1 mAbs. Both PD-1 mAbs treatment and Tim-3 deficiency in Treg cells resulted in reduced tumor growth as expected (Fig. 6A and Fig. S6A). PD-1 mAbs treatment in $T^{T /} F^{C r e}$ mice produced much greater antitumor efficacy with 3 out of 7 had complete regression compared to no completed 
regression in all the control groups (Fig. 6A and Fig. S6A). Similar to what we observed using MC38 model, we also observed synergy between Tim-3 Treg deficiency and PD-1 mAbs in B16 tumor model (Fig. S6D). In addition, using Tim-3 ${ }^{f / f}$ $F^{\text {CreErt2 }}$ mice, we also found that inducible Tim-3 deletion resulted in slower tumor growth and PD-1 mAbs treatment led to further slowed tumor growth (Fig.6B). Consistent with reduced tumor growth, flow cytometry also showed higher number or percentages of $\mathrm{CD} 45^{+}$lymphocytes and IFN- $\gamma^{+} \mathrm{CD} 4^{+} \mathrm{T}$ cells in tumors isolated from $T^{f /-} F^{C r e}$ mice treated with PD-1 mAbs compared to IgG control. (Fig. 6B-C and Fig. S6B-C). The percentage of $\mathrm{Gzmb}^{+} \mathrm{CD} 8^{+} \mathrm{T}$ cells showed a trend of increase after PD1 mAbs treatment in $T^{T /} F^{C r e}$ mice (Fig. $6 B-C$ ). These results indicate that Tim- 3 and PD-1 synergy in antitumor immune responses can be mediated by blockade of Tim-3 function on Tregs.

\section{Discussion:}

In this study, we have revealed a critical functional role Tim-3 plays in tissue Tregs.

Our data indicate that Tim-3 is important for suppressor activities of tumor tissue

Tregs. The predominant expression Tim-3 in tissue Tregs is crucial for preventing autoimmunity in neonate mice and maintaining the immune suppressive TME in adult mice. In addition, Tim-3 is required for upregulating effector molecules such as chemokines and immune inhibitory molecules of tissue Tregs. Finally, the enhanced 
antitumor activities after combination of Tim-3 deficiency in Tregs and PD-1 blockade illustrate a new mechanism of synergy between Tim3 and PD-1 blockade.

The exact role of Tim3 in the T cell-mediated immune response has been complicated due to lack of clear understanding of its unique function in different $\mathrm{T}$ cell subsets. Besides conventional T cells, Tim-3 has recently been found highly expressed on tumor and normal tissue Treg cells $(2,14-16)$. Our finding that Tim-3 plays a critical role in tissue Tregs help explain the cellular mechanisms of many previous observations. One study shows that in a GVHD model, Tim-3 is induced on activated T cells. Tim-3 blockade by a Tim-3-Ig fusion protein or Tim-3 genetic deletion in donor T cells led to increased lethality(16). These data suggest that Tim-3 signaling inhibits GVHD. Although the authors have also showed in the same study that the effect of Tim-3 blockade on GVHD is dependent on donor Treg cells, they conclude that Tim-3's function in promoting cell death in Th1 cells is responsible for its suppressive effect on GVHD. However, in light of our current finding, it is also possible that Tim-3 is required for Treg function during GVHD and the lack of Tim-3 signaling in Tregs has led to an increase in GVHD. In another study, Tim-3 is shown to inhibit alloimmune responses (43). The authors have indicated that the effect of Tim-3 blockade in preventing transplantation tolerance was mediated by inhibiting immunosuppressive function of the alloantigen-primed Tregs (43). Although the authors of this study did not pinpoint activated Tregs as the cellular source of Tim-3, 
their result is consistent with the conclusion of our study. Therefore, many studies support the notion that Tim-3 mediates immune suppression through its direct functional effect on effector Treg cells.

Our previous study demonstrates that Tim-3 is specifically up-regulated in tumor infiltrating but not peripheral Treg cells (14). Many studies also show that Tim-3 is expressed on normal tissue Treg cells (2). In addition, Tim- $3^{+}$Tregs were found to have effector phenotype and higher suppressor activities (3, 4, 15-19). In addition, combined treatement of tumor-bearing mice with PD-1 mAbs and Tim-3 mAbs led to a decrease in Treg function along with increased effector T cell function (17). Alought this finding is consistent with the idea that Tim-3 and PD-1 in combination increases the function of tumor Treg, the question remains whether Tim-3 alone is important for the function of effector Treg cells. The current study, utilizing Treg-specific Tim-3 deficient mice, demonstrates that Tim-3 is indeed required for the suppressor function of tissue Treg cells. The fact that Tim-3 deficiency results in loss of effector phenotype indicates that Tim-3 is key signaling molecule controlling the genetic programming the network of suppressive effector genes.

It has been demonstrated that PD-1 and Tim-3 mAbs have synergistic antitumor activities in many preclinical models $(12,13)$. PD-1 and Tim-3 coexpression has been considered makers of exhausted CD8 ${ }^{+} \mathrm{T}$ cells and therefore PD-1 and Tim-3 
blockade has been thought to reverse $\mathrm{CD}^{+} \mathrm{T}$ cell exhaustion $(12,13)$. Although PD$1 \mathrm{mAbs}$ has been shown to reverse $\mathrm{T}$ cell exhaustion, the role of Tim- 3 in CD8 ${ }^{+} \mathrm{T}$ cells has been controversial (44-48). In fact, blockade of Tim-3 has been shown to promote or inhibit $\mathrm{CD}^{+} \mathrm{T}$ cell immune responses depending on the experimental systems $(5,12,13,44,45,48-50)$. However, our study indicates that at least one way of synergy of PD-1 and Tim-3 blockade is through their direct function on Treg cells. Our study offers a simpler explanation for the synergy between PD-1 and Tim-3 blockade which is through a reduction of Treg function by Tim-3 mAbs and a decrease of $C D 8^{+} \mathrm{T}$ cell exhaustion by PD-1 mAbs. By providing a new mechanism of synergy between PD-1 and Tim-3 blockade, our study will shed light on targeting Tim-3 for cancer immune therapy.

\section{Material and Method}

\section{Animals}

C57BL/6, Foxp3 $3^{\text {YFP-Cre }}$ and Foxp3 $3^{\text {CreEr2GFP }}$ mice were purchased from the Jackson Labs and maintained at the specific pathogen-free animal facility of University of Pittsburgh. Tim $-3^{\text {flox/+ }}$ and Tim $-3^{+/-}$mice were generated by Transgenic and Gene Targeting (TGT) Core of University of Pittsburgh. The Crispr/Cas9 technology was used to target exon 2 of mouse Tim-3 gene with Tim-3 exon 2 floxed or deleted. Tim$3^{\text {floxflox }}$ Foxp $3^{\text {YFP-Cre }}\left(T^{\text {f/f }} F^{\text {Cre }}\right)$, Tim- $3^{+/-}$Foxp $3^{\text {YFP-Cre }}\left(T^{+/-} F^{\text {Cre }}\right)$ and Tim- $3^{\text {flox/- } F o x p} 3^{\text {YFP-Cre }}$ $\left(T^{f /-} F^{C r e}\right)$ mice were generated by crossing Foxp $3^{\text {YFP-Cre }}$, Tim $-3^{\text {flox/+ }}$ mice and Tim $-3^{+/-}$ mice for more than 5 generations. Floxed or deleted Tim-3 was confirmed by PCR and expression of Tim-3 on tumor infiltrating Treg cells were confirmed by flow 
cytometry. Tim- $3^{\text {flox/+ }}$ mice were crossed with Foxp $3^{\text {CreErt2GFP }}$-Rosa ${ }^{\text {tdTomato }}$ to generate Tim-3 $3^{\text {floxflox }}$ Foxp $3^{\text {CreErt2GFP }}-$ Rosa ${ }^{\text {tdTomato }}$ (called Tim- $3^{\text {fff }} F^{\text {CreErt2 }}$ ). All animal experiments have been approved by Institute of Animal Care and Use Committee of University of Pittsburgh.

\section{Cell lines}

Mouse colorectal cancer cell line MC38 was a generous gift from Dr. Zongsheng Guo from UPMC Hillman Cancer Center and cultured in DMEM medium supplemented with $10 \%$ fetal bovine serum and $1 \%$ penicillin-streptomycin. Mouse melanoma cell line B16 cells were cultured in RPMI-1640 medium supplemented with $10 \%$ fetal bovine serum and $1 \%$ penicillin-streptomycin.

\section{Antibodies}

For animal treatment, anti-Tim-3 (RMT3-23), anti-PD-1 (J43), polyclonal American Hamster $\lg G$ and rat $\lg G 2 a(2 A 3)$ were purchased from Bioxcell.

For flow cytometry, Ghost dye was purchased from TONBO Biosciences; anti-mouse CD45 (30-F11), anti-mouse CD4 (GK1.5), anti-mouse CD4 (RM4-5), anti-mouse CD8a (53-6.7), anti-mouse Foxp3 (MF23), anti-mouse Foxp3 (MF-14), anti-mouse Tim-3 (5D12), anti-mouse Tim-3 (RMT3-23), anti-mouse PD-1 (J43), anti-mouse PD1 (29F.1A12), anti-mouse CD62L (MEL-14), anti-mouse CD44(IM7), anti-mouse IL7R(SB/199), anti-mouse Ki67(SolA15), anti-mouse IFN-y (clone: XMG1.2), and anti-human/mouse Granzyme B (QA16A02) were purchased from BD Bioscience, Biolegend or ebioscience.

\section{Tumor Models}

6 8-week-old $T^{+/-} F^{\text {Cre }}$ and $T^{\text {t/ }} F^{\text {Cre }}$ mice were inoculated with 1 million MC38 cells or 0.1 million B16 cells intradermally (i.d.). Tumor sizes were measured every 2-3 days 
by caliper. For anti-PD-1 treatment, 6 8-week-old $T^{+/} F^{C r e}$ and $T^{f /-} F^{C r e}$ mice were inoculated with 1 million MC38 cells, when tumor sizes reached about $6 \mathrm{~mm}$ in diameter ( day 8), mice were injected intraperitoneally (i.p.) with $200 \mu$ g anti-PD-1 mAbs or lgG isotype per mouse, every 4 days for a total of 5 times. Tumor volume was calculated as $L \times W^{2} / 2$. Mice were humanely euthanized when tumor diameter reached about $20 \mathrm{~mm}$.

6 8-week-old Tim-3 ${ }^{\text {fff }} F^{\text {CreErt2 }}$ and control $F^{\text {CreEt2 }}$ mice were were injected intraperitoneally (i.p.) with tamoxifen $(50 \mathrm{mg} / \mathrm{kg}$, dissolved in ethanol/sunflower seed oil, per mouse, per dose,) every other day for a total of 7 times. 1 million MC38 cells were injected i.d. 1 day after the third tamoxifen injection. For mAbs administration, PD-1 mAbs were given when tumor diameter reached about $6 \mathrm{~mm}$ in diameter. Mice were injected intraperitoneally (i.p.) with $200 \mu \mathrm{g}$ anti-PD-1 mAbs or lgG isotype per mouse, every 4 days for a total of 4 times. Tumor volume was calculated as $L \times W^{2} / 2$. Mice were humanely euthanized when tumor diameter reached about $20 \mathrm{~mm}$.

\section{Histology}

Tim-3 $3^{\text {floxflox }}$ Foxp $3^{\text {YFP-Cre }}$ and control mice were humanely euthanized. Skin and Lung were isolated and fixed in $10 \%$ buffered formalin for 24 hours and then placed in microcassettes and fixed for another 24 hours. Tissues were then embedded in paraffin wax according to standard procedures. $5 \mu \mathrm{m}$ sections were obtained and stained with hematoxylin and eosin according to standard procedures. Tissues were scored for histopathology. Final score represents the average score of 5 different 
$\times 200$ fileds of per tissue, per mouse. Skin inflammation was scored as following: (0) no inflammation and no excessive infiltration; (1) mild inflammation and mild infiltration; (2) moderately severe inflammation and moderate infiltration; (3) severe inflammation and large infiltration of cells; (4) very severe inflammation and severe infiltration. Lung inflammation was scored as following: (0) no filtration; (1) few inflammatory cells; (2) a ring of inflammatory cells one cell layer deep; (3) a ring of inflammatory cells two to four cell layers deep; (4) a ring of inflammatory cells more than four cell layers deep.

\section{Tissue Processing and Flow Cytometry}

For skin and lung, tissues were isolated from 10 - or 18 -days old $T^{+/+} F^{\text {Cre }}$ and $T^{\text {f/f }} F^{\text {Cre }}$ neonates. For tumors, mice were injected with 1 million MC38 or 0.1 million B16 cells tumor cells i.d., when control tumors reached about $20 \mathrm{~mm}$ in diameter, mice were sacrificed and tumors were isolated. For early stage tumors, mice were injected with 1 million MC38 cells, when control tumors reached about $8 \mathrm{~mm}$ in diameter, mice were sacrificed and tumors were isolated. For mice treated with mAbs, C57BL/6 mice were inoculated with 1 million MC38 tumor cells. when tumor sizes reached about $6 \mathrm{~mm}$ in diameter, mice were injected i.p. with Anti-Tim-3 mAbs or IgG isotype. 24 hours later, mice were sacrificed and tumors were isolated. Or mice were inoculated with 1 million MC38 tumor cells, when tumor sizes reached about $6 \mathrm{~mm}$ in diameter, mice were injected intraperitoneally (i.p.) with $200 \mu$ g anti-PD-1 mAbs or lgG isotype per mouse, every 4 days for a total of 2 times or 5 times. 72 hours later, mice were sacrificed and tumors were isolated. Isolated tissues and tumors were digested in $0.25 \mathrm{mg} / \mathrm{ml}$ Liberase TL (Roche) and $0.33 \mathrm{mg} / \mathrm{ml}$ Dnase I (Sigma) and then grinded through a $40 \mu \mathrm{m}$ cell strainer to obtained single cell suspensions. For IFN- $y$ staining, single cells were first stimulated for 4 hours with $50 \mathrm{ng} / \mathrm{ml}$ phorbol 12 -myristate 13 acetate (PMA, Sigma) and $1 \mu \mathrm{g} / \mathrm{ml}$ ionomycin (Sigma) in the presence of $10 \mu \mathrm{g} / \mathrm{ml}$ Brefeldin A (Sigma). After stimulation, cells were stained for ghost dye (TONBO 
Biosciences) and antibodies against cell surface markers subsequently, followed by fixation and permeabilization with Intracellular Fixation \& Permeabilization buffer (eBioscience) according to the manufacturer's instructions. Then cells were stained with antibodies against intracellular markers. All the samples were applied to LSRII, LSRFortessa (BD Biosciences) or Aurora (Cytek Biosciences) and analyzed using Flowjo software (Tree star).

\section{The enzyme-linked immunospot (ELISpot) assay}

$15 \mu \mathrm{g} / \mathrm{ml}$ anti-IFN-Y (AN18, MabTech) was coated and incubated overnight at 4 degrees. On the other day, mice were sacrificed and spleens were isolated. $5 \times 10^{5}$ splenocytes were stimulated with $5 \times 10^{4} 200 \mathrm{~Gy}$ irradiated MC38 cells for $48 \mathrm{~h}$ in complete medium at 37 degrees. $48 \mathrm{~h}$ later, plate was washed and incubated with 1.5 $\mu \mathrm{g} / \mathrm{ml}$ biotinylated secondary antibody (R4-6A2, MabTech) and then washed and incubated with VECTASTAIN Elite ABC HRP Kit (Vector Labs) and developed with AEC Peroxidase (HRP) Substrate Kit (Vector Labs). Plate was scanned and counted using the ImmunoSpot Analyzer (Cellular Technology).

\section{Microsuppression Assay}

Splenic $C D 4^{+} C D 25^{-} \mathrm{T}$ cells were FACS sorted from naïve mouse as responder cells and labeled with $5 \mu \mathrm{M}$ CellTrace Violet (Invitrogen). FACS-sorted CD4-CD8 ${ }^{-}$ splenocytes were irradiated with 3000 rad and used as antigen presenting cells (APC). Tumoral Tregs were sorted from $T^{+/-} F^{C r e}$ and $T^{f /-} F^{\text {Cre }}$ tumor bearing mice. $5 \times 10^{3}$ responder cells and $2.5 \times 10^{4} \mathrm{APCs}$ were incubated with different number of Tregs (responder: Treg =2:1 64:1) in the presence of $2 \mu \mathrm{g} / \mathrm{ml}$ anti-CD3 $(2 \mathrm{C} 11$, Bioxcell) in a 96-well round bottom plate. $72 \mathrm{~h}$ later, cells were stained for CD4 and CD8 flow antibodies and applied to FACS analyzer. Division index were analyzed by Flowjo based on the division of the violet dye. Suppression percent was calculated as $\left(1-D I_{\text {Treg }} / D I_{\text {control }}\right) \times 100 \%$, in which $\mathrm{DI}_{\text {Treg }}$ is the division index of responder cells co- 
cultured with Tregs and $\mathrm{DI}_{\text {control }}$ is the division index of responder cells co-cultured without Tregs.

\section{RNA-seq profiling and gene expression analysis}

$1 \times 10^{3}$ Tregs (live CD $45^{+} \mathrm{CD} 4^{+} \mathrm{CD} 8^{-} \mathrm{YFP}^{+}$) were sorted by cell sorter (BD FACS Aria II) from $T^{+/-} F^{C r e}$ and $T^{f /-} F^{C r e}$ MC38 tumor-bearing mice $(n=3)$. cDNAs were prepared using the Smart-seq HT kit (Takara) and libraries were generated using the Nextera XT DNA Library Preparation kit (Illumina). Libraries were normalized, diluted and heat-denatured and then loaded for sequencing. Cluster generation and $75 \mathrm{bp}$ pairedend dual-indexed sequencing was performed using the NextSeq® 500/550 High Output Kit v2 on the NextSeq 500 Sequencing System (Illumina) in the Health Sciences Sequencing Core (HSSC) at UPMC Children's Hospital of Pittsburgh. RNA-seq data were aligned to mouse reference genome GRCm38 using STAR. Gene expression level was quantified and count expression matrices were generated using RSEM from aligned reads. Count per million (TPM) was used for further analysis. Package limma in R (51) was used to identify differentially expressed genes (DEGs) between $T^{+/-} F^{C r e}$ and $T^{f /-} F^{C r e}$ tumoral Tregs. DEGs with Benjamini-Hochberg adjusted $P$ value $<0.05$ and absolute $\log _{2}$ (fold change) $\left(\log _{2} \mathrm{FC}\right) \geq 0.5$ were selected for further analysis. The signal-to-noise ratio was used for gene ranking and javaGSEA (52) was used to run GSEA with 1000 permutations to estimate $P$ values. Corrections for multiple tests were applied using the Benjamini-Hochberg procedure. The GSEA maps were drawn by clusterProfiler(53) package in R. Heatmap figures were generated using the pheatmap package in $\mathrm{R}$. All analyses were done in $\mathrm{R}$ v.3.6.0. The application Cluego (54) in Cytoscape v.3.7.1 was used for pathway enrichment analysis of DEGs.

\section{Statistical analysis}

Student's $t$-test and Log-rank test were performed with Graphpad Prism software, $P<0.05$ was considered to be statistically significant. 
Author contributions: M.Y.and S.W devised and conducted experiments and analyzed data, wrote manuscript. C.H., Y.Z., W.Z., B.C., J.X. and W.Z. helped with experiments. Y.Y., S.L. and D.Y., helped analyze the RNA sequencing data. B.L. conceptualized and directed this study and wrote manuscript.

Acknowledgement: We thank Amanda Lu for critical reading of the manuscript. This study is partly supported by University of Pittsburgh Startup fund to B.L., R21CA2165743 to B.L., and RPCI-UPCI Ovarian Cancer SPORE DRP.

Competing interests: B.L. serves as a scientific adviser and owns stocks of Anwita Biosciences Inc. Other authors declare no competing interests.

Data and materials availability: RNA sequencing data have been deposited in the Gene Expression Omnibus under the accession number GSE140826.

\section{Figure Legend}

Figure 1. Tim-3 deficiency in Treg cells led to severe autoimmune disease.

(A) Tim-3 expression in CD4 ${ }^{+} \mathrm{T}$ cells (gated on $\mathrm{CD} 45^{+} \mathrm{CD} 4^{+}$lymphocytes) from spleen, LN and skin from 18 days-old $T^{+/+} F^{\text {Cre }}$ neonates were analyzed by flow cytometry. Data were representatives of 4-6 mice. (B) Survival curves of $T^{f / f} F^{C r e}$ and $T^{+/+} F^{\text {Cre }}$ mice ( $\mathrm{n}=12$ and 13 , respectively), Log-rank test was performed. (C-D) Representative H\&E staining pictures (C) and histology scores (D) of skin and lung (100x) from 18 days-old $T^{f / f} F^{C r e}$ and $T^{+/+} F^{C r e}$ neonates. Data were presented as 
mean \pm SEM, $n=6,{ }^{* * * *} P<0.0001$, Student's $t$ test. Pictures were representatives of 6 mice. (E-H) Tissues from 18 days-old $T^{\text {f/f }} F^{\text {Cre }}$ and $T^{+/+} F^{\text {Cre }}$ neonates were analyzed by flow cytometry. (E-F) Percentages of Treg cells (gated on CD45 $5^{+}$from spleen, $\mathrm{LN}$ and skin. Data were presented as mean \pm SEM, $n=3-5,{ }^{*} P<0.05,{ }^{* *} P<0.01$, Student's $t$ test. Flow plots $(\mathrm{G})$ and statistics $(\mathrm{H})$ of $\mathrm{CD} 45^{+}, \mathrm{CD}^{+}$and $\mathrm{CD} 8^{+}$lymphocytes, and expression of CD44, CD62L and Ki67 on Foxp3 ${ }^{-} \mathrm{CD} 4^{+}$and $\mathrm{CD} 8^{+} \mathrm{T}$ cells in skin. Data were presented as mean \pm SEM, $\mathrm{n}=3-5,{ }^{*} P<0.05,{ }^{* *} P<0.01,{ }^{* * *} P<0.001,{ }^{* * *}$ $P<0.0001$, Student's $t$ test.

Figure 2. Loss of Tim-3 in Tregs resulted in abnormal T cell activation in neonates. (A-B) Skin lymphocytes from 10 days-old $T^{\text {fff }} F^{\text {Cre }}$ and $T^{+/+} F^{C r e}$ neonates were analyzed by flow cytometry. Percentages of CD45 lymphocytes and Treg cells, Tim-3 expression on Treg cells, and expression of CD44, CD62L, IL7R and Ki67 on Foxp3 ${ }^{-} \mathrm{CD} 4^{+}$and $\mathrm{CD} 8^{+} \mathrm{T}$ cells in skin were shown. Data were presented as mean \pm SEM, $\mathrm{n}=3-5,{ }^{*} P<0.05,{ }^{* *} P<0.01,{ }^{* * *} P<0.001,{ }^{* * *} P<0.0001$, Student's $t$ test.

Figure 3. Tim-3 deletion in Treg enhanced antitumor responses and inhibited tumor growth in mice.

(A) Survival curves of $T^{\text {t/ }} F^{\text {Cre }}$ and $T^{+/+} F^{\text {Cre }}$ mice ( $n=20$ and 13, respectively), Log-rank test. (B) Growth curves of MC38 tumors in $T^{4 /-} F^{C r e}$ and $T^{+/-} F^{C r e}$ mice. Data were presented as mean \pm SEM, $n=5$, Student's $t$ test. (C) Growth curves of MC38 tumors 
in $T^{f / f} F^{\text {CreErt2 }}$ and $F^{\text {CreErt2 }}$ mice. Data were presented as mean \pm SEM, $\mathrm{n}=5$, Student's $t$ test. (D-F) MC38 tumors (6 8 $\mathrm{mm}$ in diameter) were analyzed by flow cytometry and splenocytes were analyzed by Elispot assay. (D-E) Percentage and total number of tumor infiltrating $\mathrm{CD} 45^{+}$lymphocytes, percentages of $\mathrm{CD} 4^{+} \mathrm{T}, \mathrm{CD} 8^{+} \mathrm{T}$ cells and Treg cells and expression of granzyme B, IFN- $\gamma$, PD-1, and Tim-3 on Foxp3-CD4 ${ }^{+}$and $\mathrm{CD}^{+} \mathrm{T}$ cells were shown. Data were presented as mean \pm SEM, $\mathrm{n}=3-4$. ${ }^{*} P<0.05$, ** $P<0.01,{ }^{* *} P<0.001$, Student's $t$ test. (F) Numbers of tumor-antigen specific IFN- $\gamma$ producers in the splenocytes were obtained by the Elispot assay $(n=3-4)$. Data were presented as mean \pm SEM, ${ }^{*} P<0.05,{ }^{* *} P<0.01,{ }^{* * *} P<0.001$, Student's $t$ test.

Figure 4. Blockade of Tim-3 function diminished the number and suppression activity of Treg cells in the TME.

(A) Tumoral Treg cells were sorted from $T^{f /} F^{C r e}$ and $T^{+/-} F^{C r e}$ MC38 tumor-bearing mice (6 8mm in diameter) and assayed in a suppression assay, data were representative of 3 independent experiments with similar results. ${ }^{*} P<0.05$, Student's $t$ test. (B-C) CD44, IL7R and PD-1 expression on tumoral Treg cells (as in A gated on $\mathrm{CD}_{4} 5^{+} \mathrm{CD}^{+} \mathrm{Foxp}^{+}$) from $T^{\mathrm{f} /-} \mathrm{F}^{\mathrm{Cre}}$ and $T^{+/-} \mathrm{F}^{\mathrm{Cre}} \mathrm{MC} 38$ tumor-bearing mice $(\mathrm{n}=3-4)$. Data were presented as mean \pm SEM, ${ }^{*} P<0.05$, Student's $t$ test. (D-E) C57BL/6 mice were inoculated with MC38 tumors ( $\sim 8 \mathrm{~mm}$ in diameter) and $\mathrm{Tim}-3^{+}$and Tim- $3^{-}$tumoral Tregs (gated on CD45 ${ }^{+} \mathrm{CD} 4^{+}$Foxp3 ${ }^{+}$) were analyzed for CD44, IL7R and PD-1 expression by flow cytometry $(n=3-4)$. Data were presented as mean \pm SEM, ** 
$P<0.01,{ }^{* * *} P<0.001$, Student's $t$ test. (F-G) C57BL/6 mice were inoculated with MC38 tumors, when diameter reached about $8 \mathrm{~mm}$, mice were treated with anti-Tim3 or IgG control. 24h later, tumoral Tregs cells were analyzed for CD44, IL7R and PD-1 expression $(n=3)$. Data were presented as mean \pm SEM, ${ }^{*} P<0.05$, ${ }^{* *} P<0.001$, Student's $t$ test.

Figure 5. Transcriptome analysis of the Tim-3-driven molecular program in tumoral Treg cells.

(A-C) Bulk RNA sequencing analysis of tumoral Tregs from $T^{f /-} F^{C r e}$ and $T^{+/-} F^{C r e}$ MC38 tumor-bearing mice $(6 \sim 7 \mathrm{~mm}$ in diameter) $(\mathrm{n}=3)$. (A) Volcano plots showing enrichment of DEGs in $T^{f /-} F^{C r e}$ and $T^{+/-} F^{C r e}$ Tregs. DEGs with Benjamini-Hochberg adjusted $P$ value $<0.05$ and absolute $\log _{2}$ (fold change) $\left(\log _{2} \mathrm{FC}\right) \geq 0.5$ were selected for further analysis. (B) GSEA analysis of DEGs enriched in $T^{\text {f/ }} \mathrm{F}^{\mathrm{Cre}}$ and $T^{+/-} \mathrm{F}^{\mathrm{Cre}}$ Tregs with indicated gene signatures. $P$ value? (C) Heatmap showing deferentially expressed genes in $T^{f /-} F^{C r e}$ or $T^{+/-} F^{C r e}$ Treg cells. The genes were selected based on functional categories and adjusted $P$ value $<0.05$ and $\log _{2} \mathrm{FC} \geq 1$.

\section{Figure 6. Tim-3 deletion in Treg cells synergized with PD-1 mAbs in tumor}

\section{immunotherapy.}

(A-B). Mean volumes of MC38 tumors treated with IgG isotype or PD-1 mAbs in $T^{+/}$ $F^{\text {Cre }}$ and $T^{f /-} F^{C r e}$ mice $(\mathrm{A}, \mathrm{n}=5-7)$ and Foxp $3^{\text {CreERT2 }}$ and $T^{\mathrm{f} / \mathrm{f}}$ Foxp $3^{\text {CreERT2 }}$ mice $(\mathrm{B}, \mathrm{n}=5$ 6). Data were presented as mean \pm SEM, ${ }^{*} P<0.05$, ${ }^{* *} P<0.01,{ }^{* * *} P<0.001$, One-way ANOVA test and Student's $t$ test. (C-D). $T^{+/-} F^{\text {Cre }}$ and $T^{\text {t/- }} F^{\text {Cre }}$ mice were inoculated 
with MC38 tumors, when tumor sizes reached $6 \mathrm{~mm}$, mice were treated with PD-1 mAbs or control lgG for a total of 2 times. 72 hours later, tumors were isolated and analyzed by flow cytometry. Representative flow plots and statistics of CD45 $5^{+}, \mathrm{CD} 4^{+}$ and $\mathrm{CD}^{+}$lymphocytes and IFN- $\gamma$, granzyme B production were showed $(n=4)$. Data were presented as mean \pm SEM, ${ }^{*} P<0.05,{ }^{* *} P<0.01$, ${ }^{* *} P<0.001$, Student's $t$ test were performed. Data are representative three independent experiments.

\section{Reference}

1. Sakaguchi S, Yamaguchi T, Nomura T, and Ono M. Regulatory T cells and immune tolerance. Cell. 2008;133(5):775-87.

2. Panduro M, Benoist C, and Mathis D. Tissue Tregs. Annu Rev Immunol. 2016;34:609-33.

3. Miragaia RJ, Gomes T, Chomka A, Jardine L, Riedel A, Hegazy AN, et al. Single-Cell Transcriptomics of Regulatory T Cells Reveals Trajectories of Tissue Adaptation. Immunity. 2019;50(2):493-504 e7.

4. Zemmour D, Zilionis R, Kiner E, Klein AM, Mathis D, and Benoist C. Singlecell gene expression reveals a landscape of regulatory $\mathrm{T}$ cell phenotypes shaped by the TCR. Nat Immunol. 2018;19(3):291-301.

5. Monney L, Sabatos CA, Gaglia JL, Ryu A, Waldner H, Chernova T, et al. Th1-specific cell surface protein Tim-3 regulates macrophage activation and severity of an autoimmune disease. Nature. 2002;415(6871):536-41.

6. Anderson AC, Lord GM, Dardalhon V, Lee DH, Sabatos-Peyton CA, Glimcher LH, et al. T-bet, a Th1 transcription factor regulates the expression of Tim-3. Eur J Immunol. 2010;40(3):859-66.

7. Hastings WD, Anderson DE, Kassam N, Koguchi K, Greenfield EA, Kent SC, et al. TIM-3 is expressed on activated human CD4+ T cells and regulates Th1 and Th17 cytokines. Eur J Immunol. 2009;39(9):2492-501.

8. Chihara N, Madi A, Kondo T, Zhang H, Acharya N, Singer M, et al. Induction and transcriptional regulation of the co-inhibitory gene module in $\mathrm{T}$ cells. Nature. 2018;558(7710):454-9.

9. Golden-Mason L, Palmer BE, Kassam N, Townshend-Bulson L, Livingston S, McMahon BJ, et al. Negative immune regulator Tim-3 is overexpressed on $\mathrm{T}$ cells in hepatitis $\mathrm{C}$ virus infection and its blockade rescues dysfunctional CD4+ and CD8+ T cells. J Virol. 2009;83(18):9122-30. 
10. Jin HT, Anderson AC, Tan WG, West EE, Ha SJ, Araki K, et al. Cooperation of Tim-3 and PD-1 in CD8 T-cell exhaustion during chronic viral infection. Proceedings of the National Academy of Sciences of the United States of America. 2010;107(33):14733-8.

11. Takamura S, Tsuji-Kawahara S, Yagita H, Akiba H, Sakamoto M, Chikaishi $\mathrm{T}$, et al. Premature terminal exhaustion of Friend virus-specific effector CD8+ $\mathrm{T}$ cells by rapid induction of multiple inhibitory receptors. Journal of immunology. 2010;184(9):4696-707.

12. Sakuishi K, Apetoh L, Sullivan JM, Blazar BR, Kuchroo VK, and Anderson AC. Targeting Tim-3 and PD-1 pathways to reverse T cell exhaustion and restore anti-tumor immunity. J Exp Med. 2010;207(10):2187-94.

13. Fourcade J, Sun Z, Benallaoua M, Guillaume P, Luescher IF, Sander C, et al. Upregulation of Tim-3 and PD-1 expression is associated with tumor antigenspecific CD8+ T cell dysfunction in melanoma patients. J Exp Med. 2010;207(10):2175-86.

14. Gao X, Zhu Y, Li G, Huang H, Zhang G, Wang F, et al. TIM-3 expression characterizes regulatory $\mathrm{T}$ cells in tumor tissues and is associated with lung cancer progression. PLoS One. 2012;7(2):e30676.

15. Liu Z, McMichael EL, Shayan G, Li J, Chen K, Srivastava R, et al. Novel Effector Phenotype of Tim-3(+) Regulatory T Cells Leads to Enhanced Suppressive Function in Head and Neck Cancer Patients. Clinical cancer research : an official journal of the American Association for Cancer Research. 2018;24(18):4529-38.

16. Veenstra RG, Taylor PA, Zhou Q, Panoskaltsis-Mortari A, Hirashima M, Flynn R, et al. Contrasting acute graft-versus-host disease effects of Tim3/galectin-9 pathway blockade dependent upon the presence of donor regulatory T cells. Blood. 2012;120(3):682-90.

17. Sakuishi K, Ngiow SF, Sullivan JM, Teng MW, Kuchroo VK, Smyth MJ, et al. TIM3 $(+)$ FOXP3 $(+)$ regulatory $\mathrm{T}$ cells are tissue-specific promoters of Tcell dysfunction in cancer. Oncoimmunology. 2013;2(4):e23849.

18. Gupta S, Thornley TB, Gao W, Larocca R, Turka LA, Kuchroo VK, et al. Allograft rejection is restrained by short-lived TIM-3+PD-1+Foxp3+ Tregs. The Journal of clinical investigation. 2012;122(7):2395-404.

19. Gautron AS, Dominguez-Villar M, de Marcken M, and Hafler DA. Enhanced suppressor function of TIM-3+ FoxP3+ regulatory T cells. Eur J Immunol. 2014;44(9):2703-11.

20. Brunkow ME, Jeffery EW, Hjerrild KA, Paeper B, Clark LB, Yasayko SA, et al. Disruption of a new forkhead/winged-helix protein, scurfin, results in the fatal lymphoproliferative disorder of the scurfy mouse. Nat Genet. 2001;27(1):68-73. 
21. Scharschmidt TC, Vasquez KS, Truong HA, Gearty SV, Pauli ML, Nosbaum A, et al. A Wave of Regulatory T Cells into Neonatal Skin Mediates Tolerance to Commensal Microbes. Immunity. 2015;43(5):1011-21.

22. Oja AE, Piet B, van der Zwan D, Blaauwgeers H, Mensink M, de Kivit S, et al. Functional Heterogeneity of CD4(+) Tumor-Infiltrating Lymphocytes With a Resident Memory Phenotype in NSCLC. Front Immunol. 2018;9:2654.

23. Downs-Canner S, Berkey S, Delgoffe GM, Edwards RP, Curiel T, Odunsi K, et al. Suppressive IL-17A(+)Foxp3(+) and ex-Th17 IL-17A(neg)Foxp3(+) Treg cells are a source of tumour-associated Treg cells. Nature communications. 2017;8:14649.

24. Wang L, Simons DL, Lu X, Tu TY, Solomon S, Wang R, et al. Connecting blood and intratumoral Treg cell activity in predicting future relapse in breast cancer. Nat Immunol. 2019.

25. Dong L, Nordlohne J, Ge S, Hertel B, Melk A, Rong S, et al. T Cell CX3CR1 Mediates Excess Atherosclerotic Inflammation in Renal Impairment. J Am Soc Nephrol. 2016;27(6):1753-64.

26. Patterson SJ, Pesenacker AM, Wang AY, Gillies J, Mojibian M, Morishita K, et al. T regulatory cell chemokine production mediates pathogenic $\mathrm{T}$ cell attraction and suppression. The Journal of clinical investigation. 2016;126(3):1039-51.

27. Ondondo B, Colbeck E, Jones E, Smart K, Lauder SN, Hindley J, et al. A distinct chemokine axis does not account for enrichment of Foxp3(+) CD4(+) $\mathrm{T}$ cells in carcinogen-induced fibrosarcomas. Immunology. 2015;145(1):94104.

28. Assas BM, Pennock JI, and Miyan JA. Calcitonin gene-related peptide is a key neurotransmitter in the neuro-immune axis. Front Neurosci. 2014;8:23.

29. Johnson DB, Nixon MJ, Wang Y, Wang DY, Castellanos E, Estrada MV, et al. Tumor-specific MHC-II expression drives a unique pattern of resistance to immunotherapy via LAG-3/FCRL6 engagement. JCI Insight. 2018;3(24).

30. Palani S, Elima K, Ekholm E, Jalkanen S, and Salmi M. Monocyte Stabilin-1 Suppresses the Activation of Th1 Lymphocytes. Journal of immunology. 2016;196(1):115-23.

31. Yang Y, Stang A, Schweickert PG, Lanman NA, Paul EN, Monia BP, et al. Thrombin Signaling Promotes Pancreatic Adenocarcinoma through PAR-1Dependent Immune Evasion. Cancer research. 2019;79(13):3417-30.

32. Rujan RM, and Reynolds CA. Calcitonin Gene-Related Peptide Antagonists and Therapeutic Antibodies. Handb Exp Pharmacol. 2019.

33. Moore KW, de Waal Malefyt R, Coffman RL, and O'Garra A. Interleukin-10 and the interleukin-10 receptor. Annu Rev Immunol. 2001;19:683-765.

34. Rexin P, Tauchert A, Hanze J, Heers H, Schmidt A, Hofmann R, et al. The Immune Checkpoint Molecule CD200 Is Associated with Tumor Grading and Metastasis in Bladder Cancer. Anticancer Res. 2018;38(5):2749-54. 
35. Andrews LP, Marciscano AE, Drake CG, and Vignali DA. LAG3 (CD223) as a cancer immunotherapy target. Immunol Rev. 2017;276(1):80-96.

36. Mycko MP, Sliwinska B, Cichalewska M, Cwiklinska H, Raine CS, and Selmaj KW. Brain glycolipids suppress T helper cells and inhibit autoimmune demyelination. J Neurosci. 2014;34(25):8646-58.

37. Yamada A, Arakaki R, Saito M, Kudo Y, and Ishimaru N. Dual Role of Fas/FasL-Mediated Signal in Peripheral Immune Tolerance. Front Immunol. 2017;8:403.

38. Takenaka MC, Robson S, and Quintana FJ. Regulation of the T Cell Response by CD39. Trends Immunol. 2016;37(7):427-39.

39. Boucard-Jourdin M, Kugler D, Endale Ahanda ML, This S, De Calisto J, Zhang A, et al. beta8 Integrin Expression and Activation of TGF-beta by Intestinal Dendritic Cells Are Determined by Both Tissue Microenvironment and Cell Lineage. Journal of immunology. 2016;197(5):1968-78.

40. Lacy-Hulbert A, Smith AM, Tissire H, Barry M, Crowley D, Bronson RT, et al. Ulcerative colitis and autoimmunity induced by loss of myeloid alphav integrins. Proceedings of the National Academy of Sciences of the United States of America. 2007;104(40):15823-8.

41. Travis MA, Reizis B, Melton AC, Masteller E, Tang Q, Proctor JM, et al. Loss of integrin alpha(v)beta8 on dendritic cells causes autoimmunity and colitis in mice. Nature. 2007;449(7160):361-5.

42. Lu B, Zagouras P, Fischer JE, Lu J, Li B, and Flavell RA. Kinetic analysis of genomewide gene expression reveals molecule circuitries that control $\mathrm{T}$ cell activation and Th1/2 differentiation. Proceedings of the National Academy of Sciences of the United States of America. 2004;101(9):3023-8.

43. Sanchez-Fueyo A, Tian J, Picarella D, Domenig C, Zheng XX, Sabatos CA, et al. Tim-3 inhibits $\mathrm{T}$ helper type 1-mediated auto- and alloimmune responses and promotes immunological tolerance. Nat Immunol. 2003;4(11):1093-101.

44. Gorman JV, and Colgan JD. Response to Comment on "Tim-3 directly enhances CD8 T cell responses to acute Listeria monocytogenes infection". Journal of immunology. 2014;193(2):467-8.

45. Gorman JV, Starbeck-Miller G, Pham NL, Traver GL, Rothman PB, Harty JT, et al. Tim-3 directly enhances CD8 T cell responses to acute Listeria monocytogenes infection. Journal of immunology. 2014;192(7):3133-42.

46. Kuchroo VK, Anderson AC, and Freeman GJ. Comment on "Tim-3 directly enhances CD8 T cell responses to acute Listeria monocytogenes infection". Journal of immunology. 2014;193(2):467.

47. Du W, Yang M, Turner A, Xu C, Ferris RL, Huang J, et al. TIM-3 as a Target for Cancer Immunotherapy and Mechanisms of Action. Int J Mol Sci. 2017;18(3).

48. Ferris RL, Lu B, and Kane LP. Too much of a good thing? Tim-3 and TCR signaling in T cell exhaustion. Journal of immunology. 2014;193(4):1525-30. 
49. Avery L, Filderman J, Szymczak-Workman AL, and Kane LP. Tim-3 costimulation promotes short-lived effector T cells, restricts memory precursors, and is dispensable for T cell exhaustion. Proceedings of the National Academy of Sciences of the United States of America. 2018;115(10):2455-60.

50. Hafler DA, and Kuchroo V. TIMs: central regulators of immune responses. The Journal of experimental medicine. 2008;205(12):2699-701.

51. Ritchie ME, Phipson B, Wu D, Hu Y, Law CW, Shi W, et al. limma powers differential expression analyses for RNA-sequencing and microarray studies. Nucleic Acids Res. 2015;43(7):e47.

52. Subramanian A, Tamayo P, Mootha VK, Mukherjee S, Ebert BL, Gillette MA, et al. Gene set enrichment analysis: a knowledge-based approach for interpreting genome-wide expression profiles. Proceedings of the National Academy of Sciences of the United States of America. 2005;102(43):15545-50.

53. Yu G, Wang LG, Han Y, and He QY. clusterProfiler: an R package for comparing biological themes among gene clusters. OMICS. 2012;16(5):284-7.

54. Bindea G, Mlecnik B, Hackl H, Charoentong P, Tosolini M, Kirilovsky A, et al. ClueGO: a Cytoscape plug-in to decipher functionally grouped gene ontology and pathway annotation networks. Bioinformatics. 2009;25(8):10913. 
Fig. 1
A Spleen

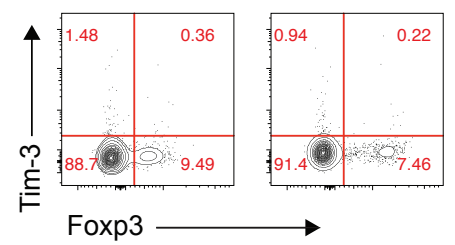

Skin $\quad$ B $\quad-T^{+/+} F^{\text {Cre }}$

C
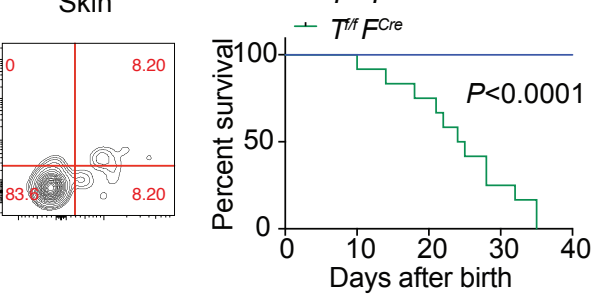

skin

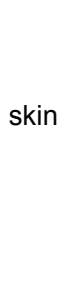

$T^{+/+} F^{\text {cre }}$

$T^{f / f} F^{C r e}$

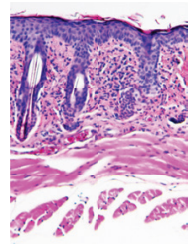

lung

$\square T^{+/+} F^{\text {Cre }}$
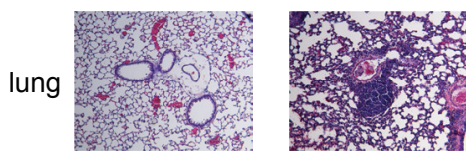

D

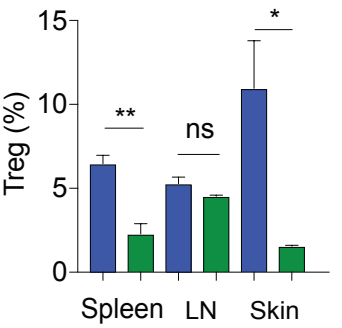

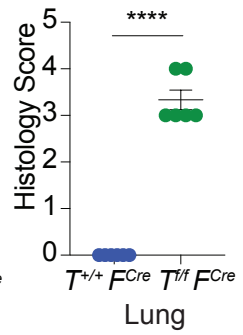

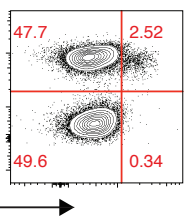

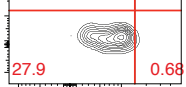

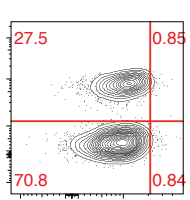

Spleen LN Skin

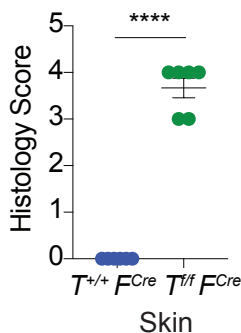

G

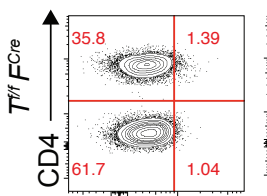

Foxp3

Foxp3-CD4 ${ }^{+}$
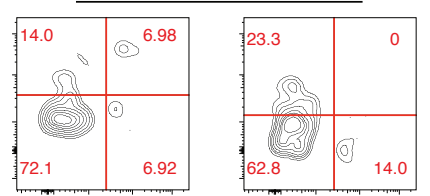

$\mathrm{CD}^{+}$
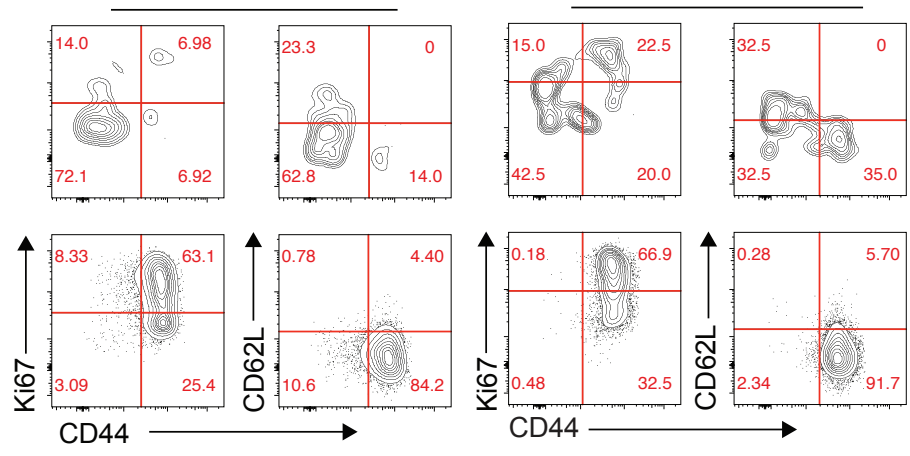

H
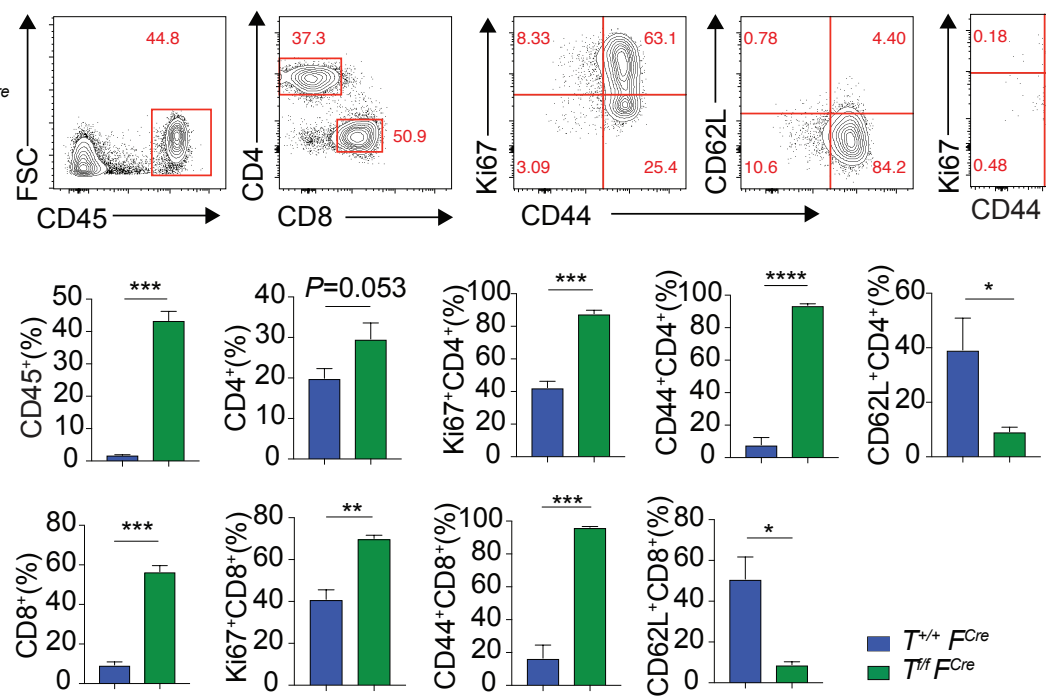

$\square T^{+/+} F^{\text {Cre }}$
$\square T^{1 / f} F^{C r e}$ 
Fig. 2

A

Live cells $\quad \mathrm{CD}^{+}$

Foxp3-CD4 ${ }^{+}$

$\mathrm{CD}^{+}$
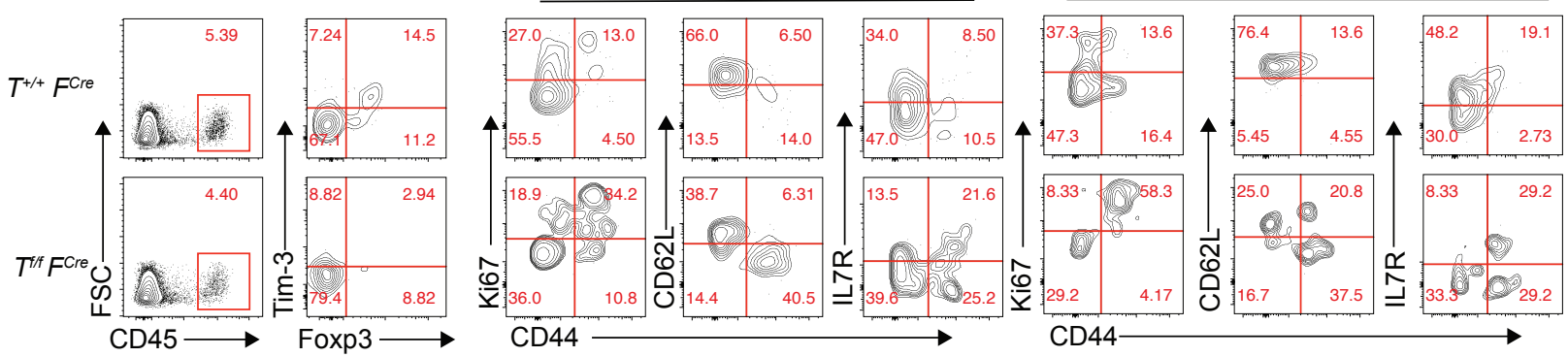

B
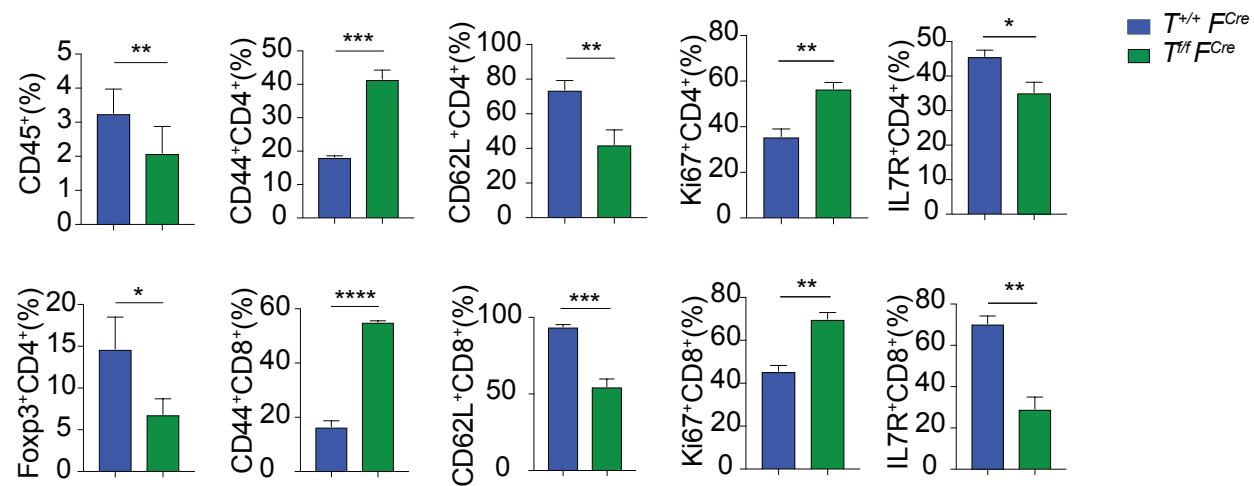
Fig. 3

A

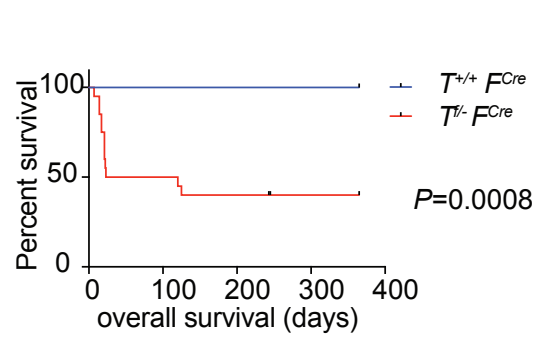

B

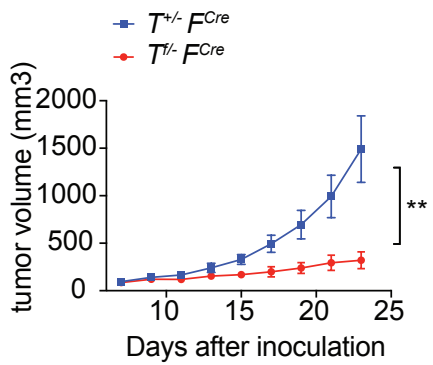

C

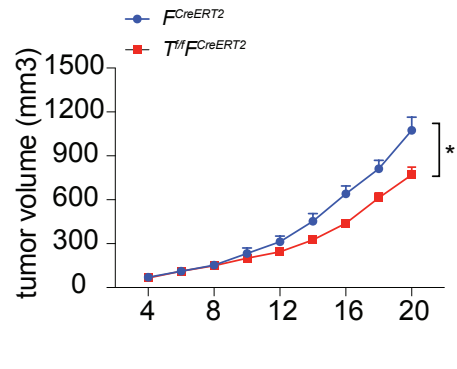

D

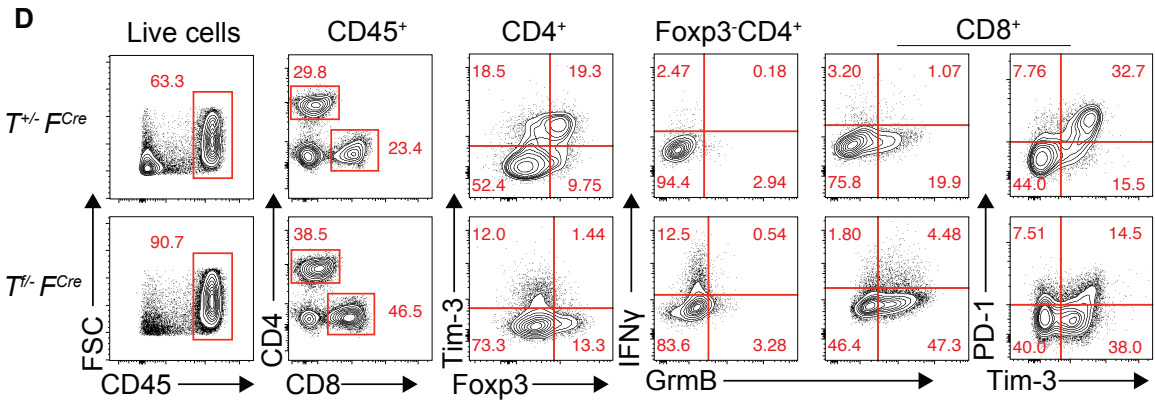

E

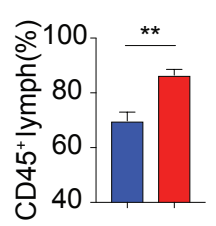

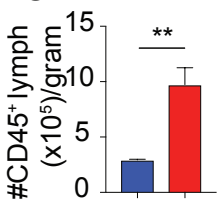
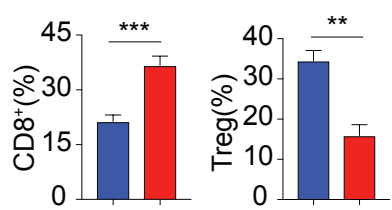

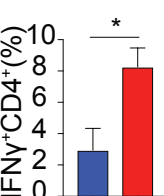

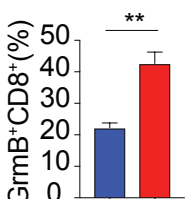

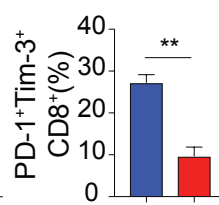

F

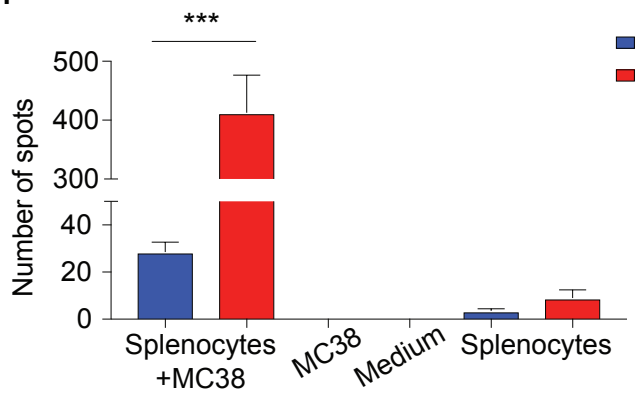

$T^{+/-} F^{C r e}$

- $T^{1 /-} F^{C r e}$ 
Fig. 4

A

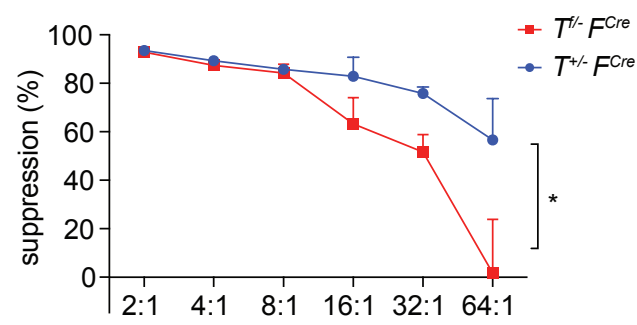

Responder : Treg

D

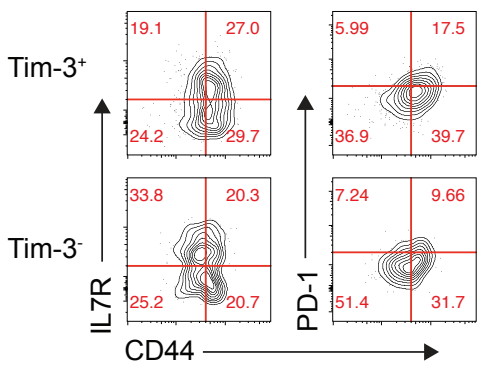

F

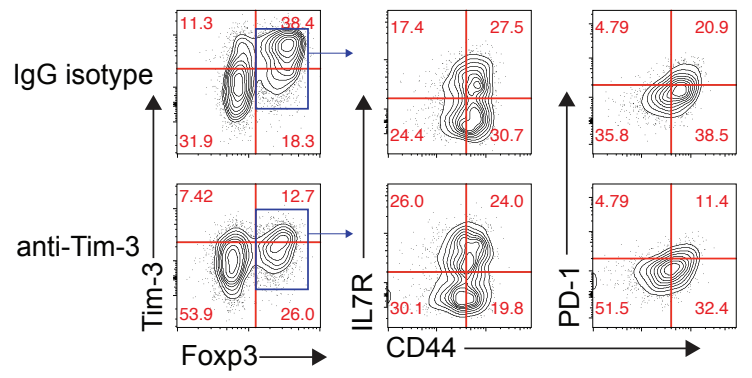

B

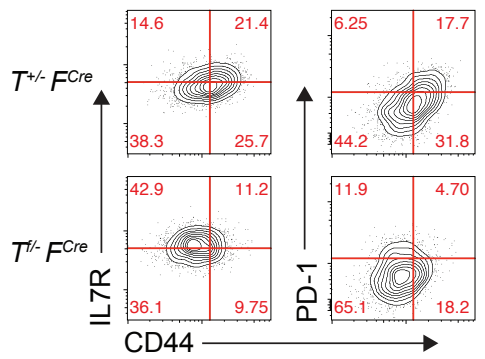

C

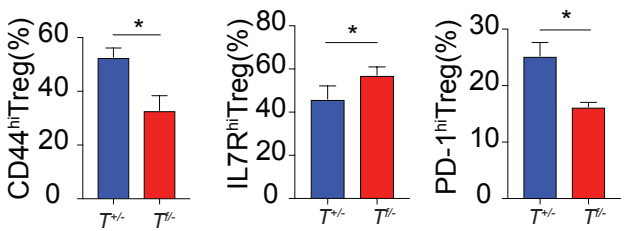

E

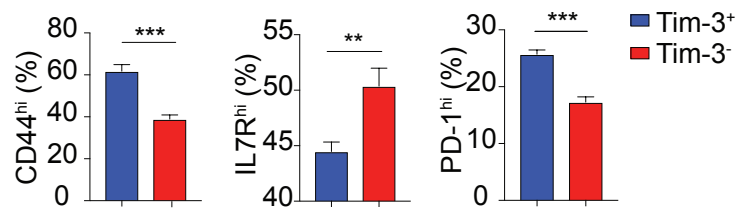

G
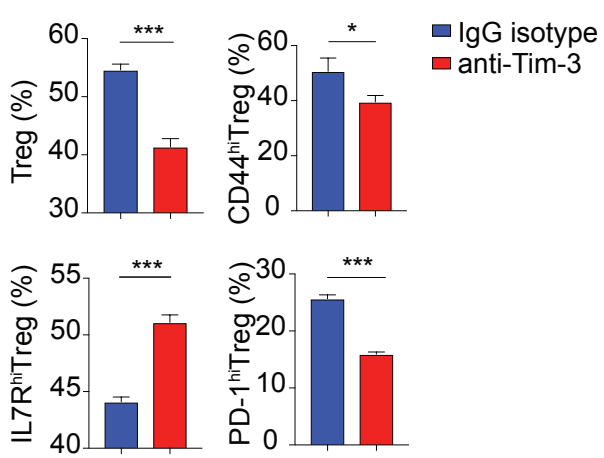
Fig. 5

A

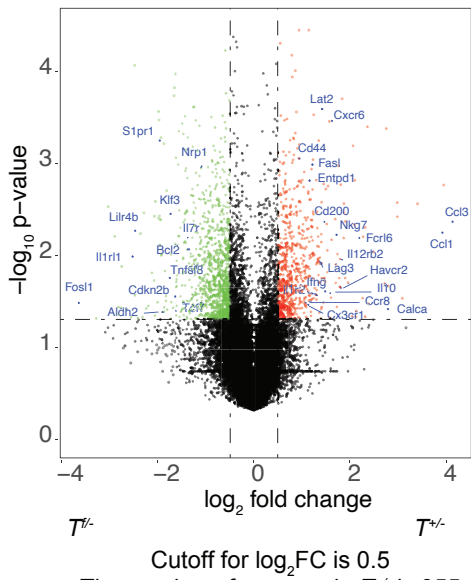

The number of up gene in $T^{+/}$is 955

The number of up gene in $T^{/ /}$is 675

\section{C}

B
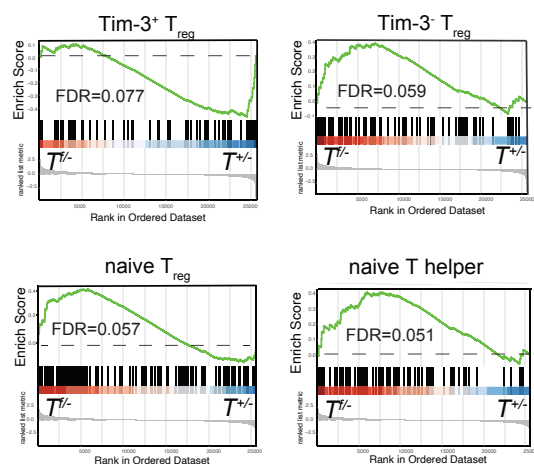

$T^{+/-} F^{C r e} \quad T^{1 /-} F^{C r e}$

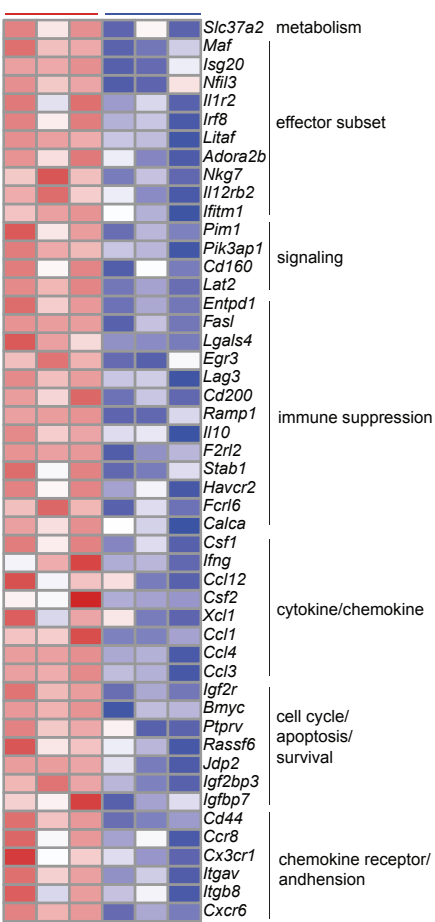

$T^{+/} F^{\mathrm{Cre}} \quad T^{1 /-} F^{\mathrm{Cre}}$

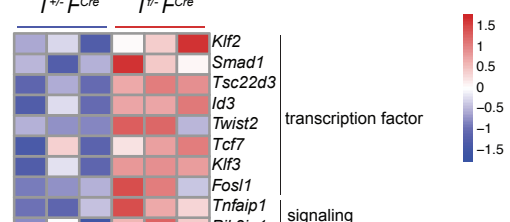


Fig. 6

A
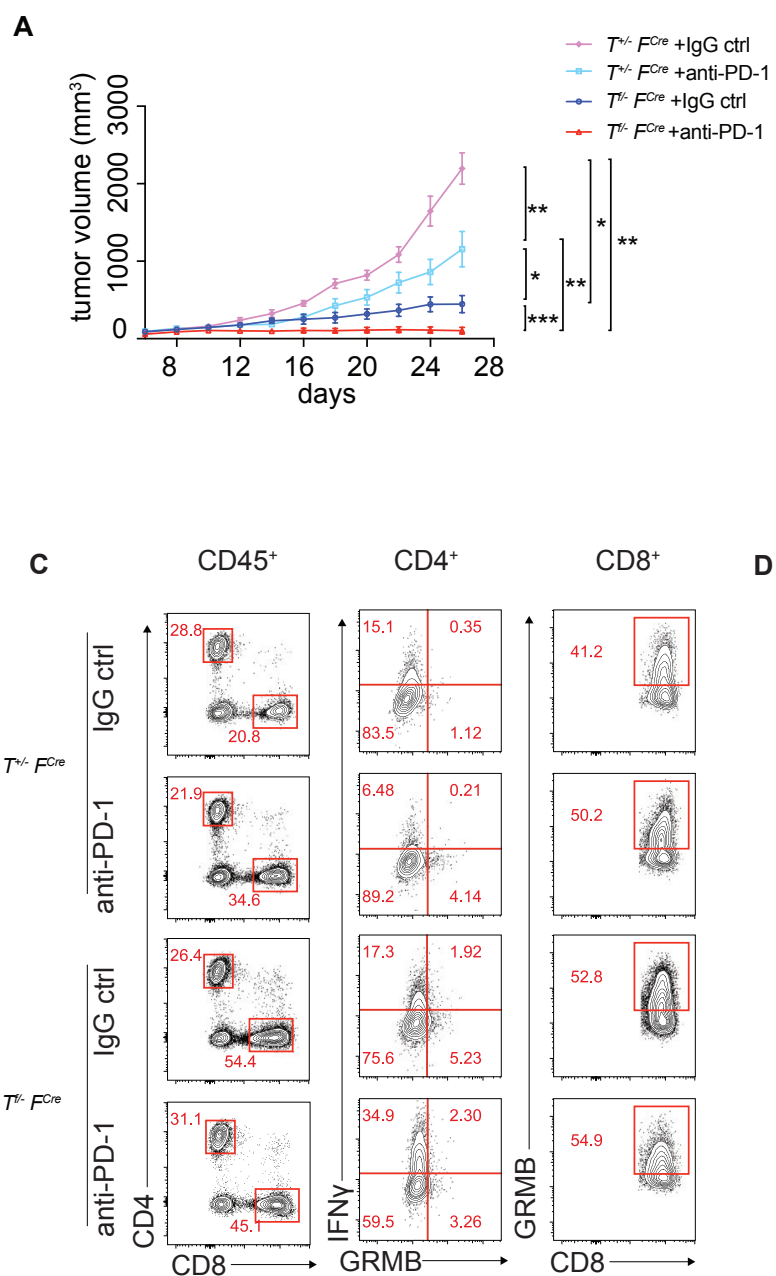

B

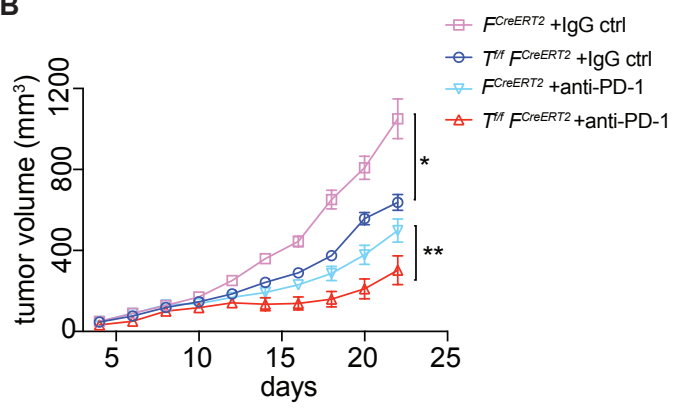

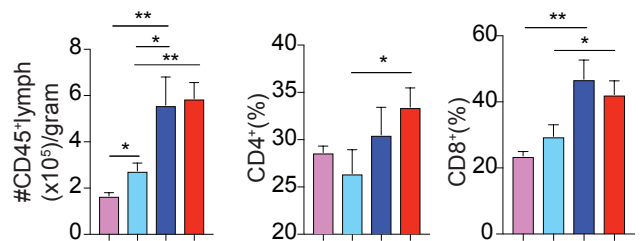

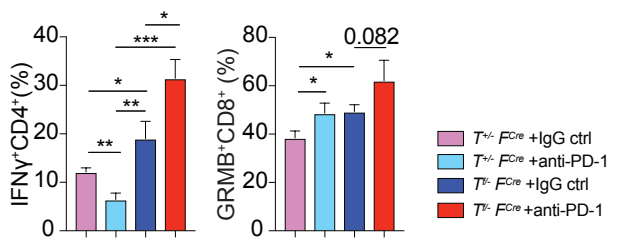


Figures

Fig. 1
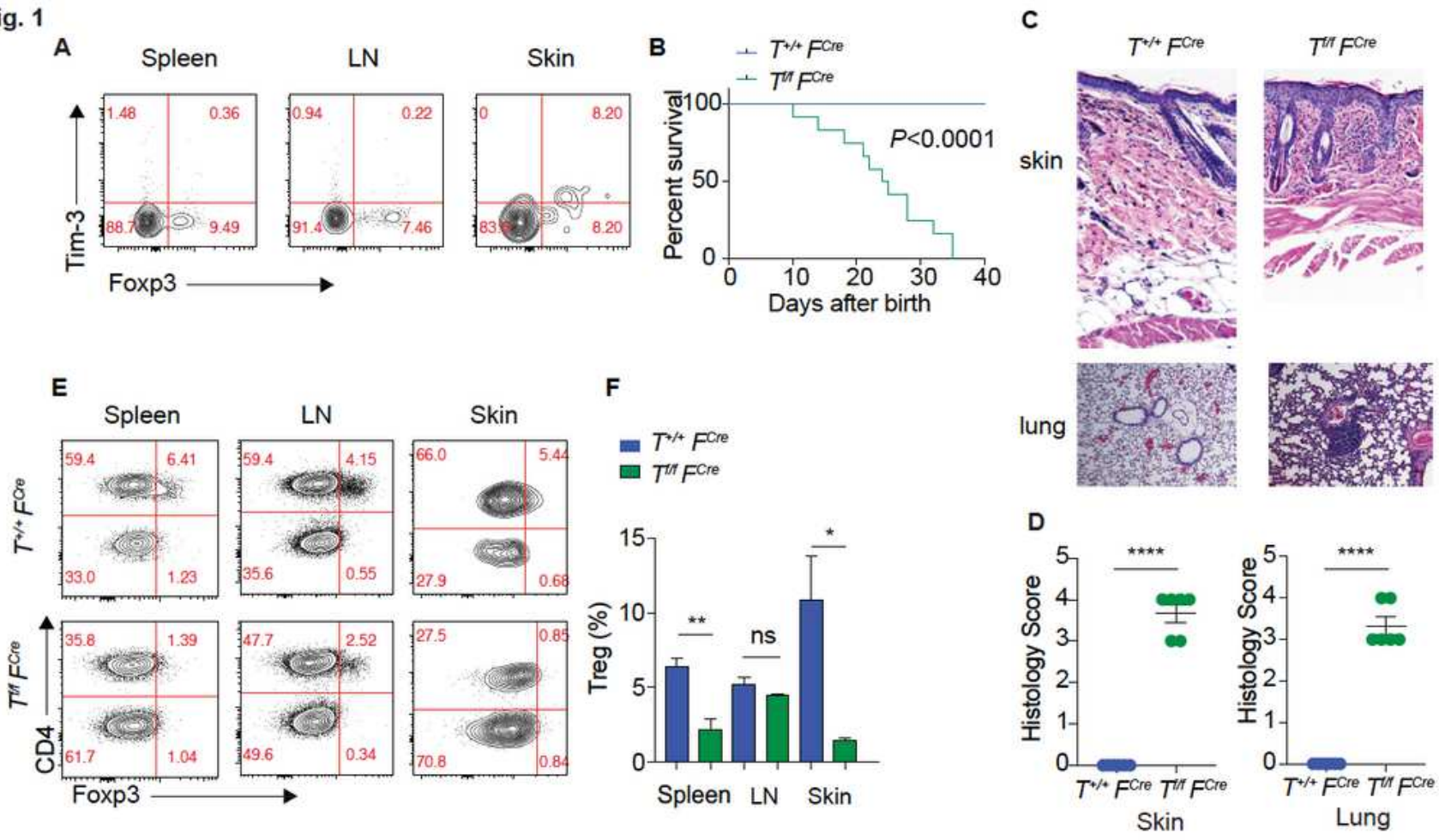

$$
\text { G }
$$
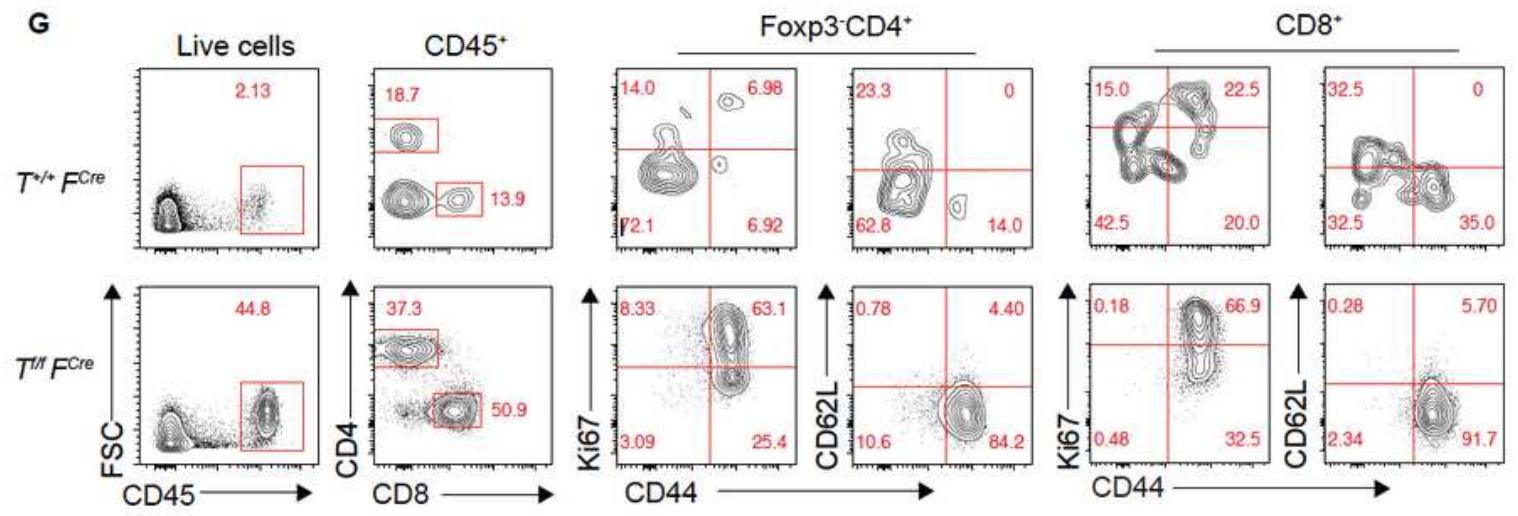

H
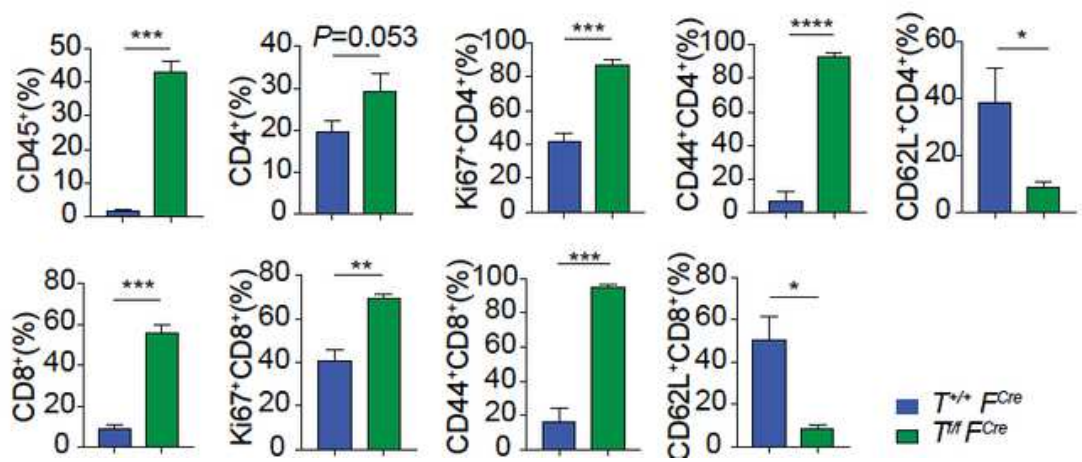

\section{Figure 1}

Tim-3 deficiency in Treg cells led to severe autoimmune disease. (A) Tim-3 expression in CD4+ T cells (gated on CD45+CD4+ lymphocytes) from spleen, $L N$ and skin from 18 days-old $T+/+F C$ re neonates were analyzed by flow cytometry. Data were representatives of 4-6 mice. (B) Survival curves of $T f / f$ FCre and 
$\mathrm{T}+/+$ FCre mice ( $\mathrm{n}=12$ and 13, respectively), Log-rank test was performed. (C-D) Representative H\&E staining pictures (C) and histology scores (D) of skin and lung (100x) from 18 days-old Tf/f FCre and $T+/+$ FCre neonates. Data were presented as mean $\pm S E M, n=6, * \star \star * P<0.0001$, Student's $t$ test. Pictures were representatives of 6 mice. $(\mathrm{E}-\mathrm{H})$ Tissues from 18 days-old Tf/f FCre and T+/+FCre neonates were analyzed by flow cytometry. (E-F) Percentages of Treg cells (gated on CD45+) from spleen, LN and skin. Data were presented as mean $\pm S E M, n=3-5, * P<0.05$, $* * P<0.01$, Student's $t$ test. Flow plots $(G)$ and statistics $(\mathrm{H})$ of CD45+, CD4+ and CD8+ lymphocytes, and expression of CD44, CD62L and Ki67 on Foxp3-CD4+ and CD8+ T cells in skin. Data were presented as mean $\pm S E M, n=3-5, * P<0.05, * \star P<0.01$, *** $P<0.001, * \star \star \star P<0.0001$, Student's t test.
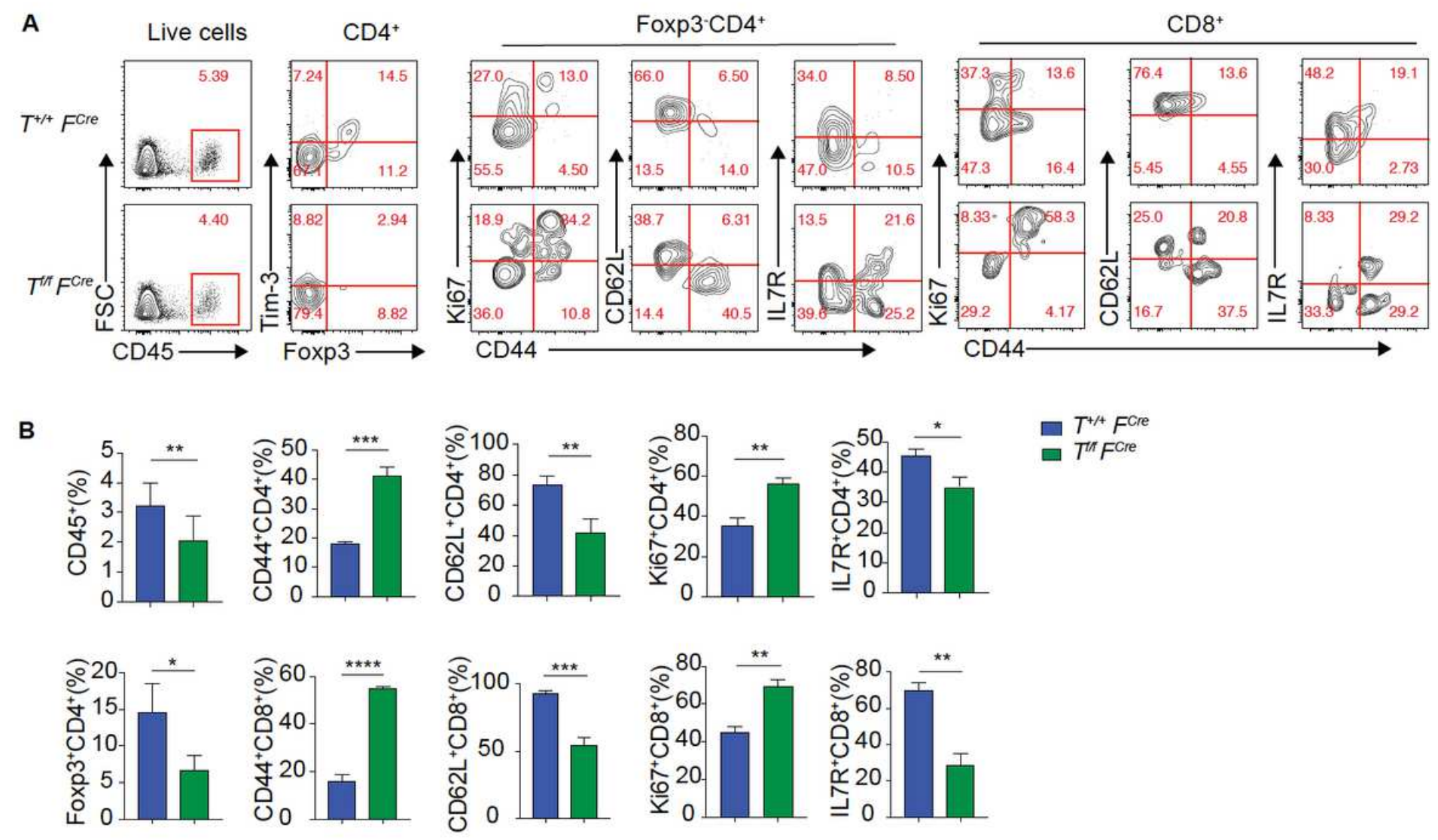

\section{Figure 2}

Loss of Tim-3 in Tregs resulted in abnormal T cell activation in neonates. (A-B) Skin lymphocytes from 10 days-old Tf/f FCre and T+/+ FCre neonates were analyzed by flow cytometry. Percentages of CD45+ lymphocytes and Treg cells, Tim-3 expression on Treg cells, and expression of CD44, CD62L, IL7R and Ki67 on Foxp3-CD4+ and CD8+ T cells in skin were shown. Data were presented as mean $\pm S E M, n=3-5$,

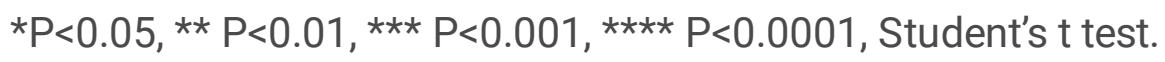


A

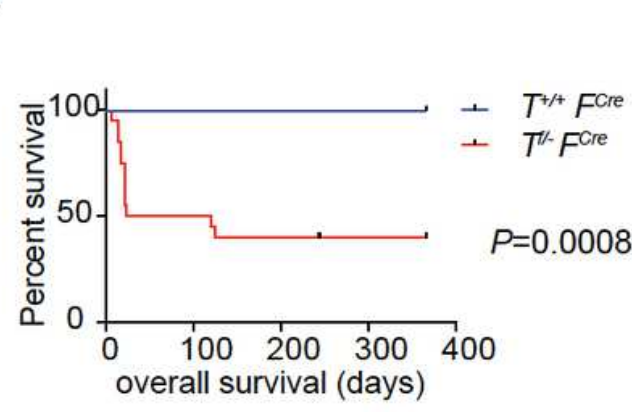

B

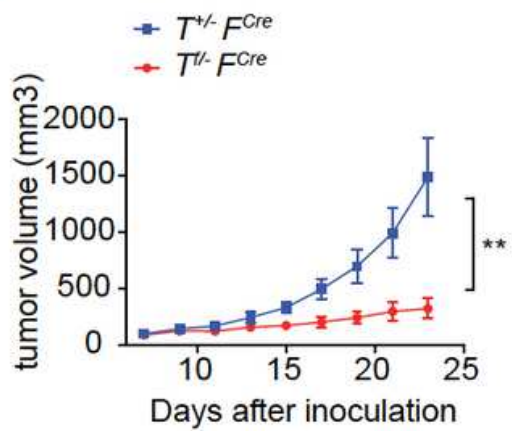

C

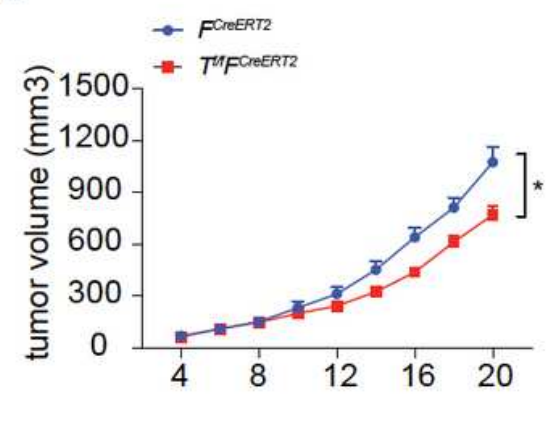

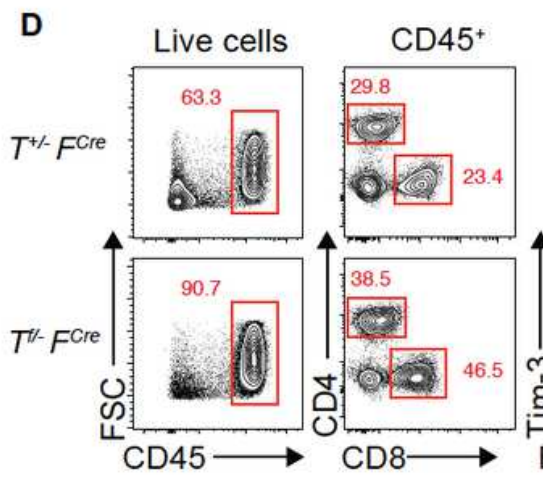
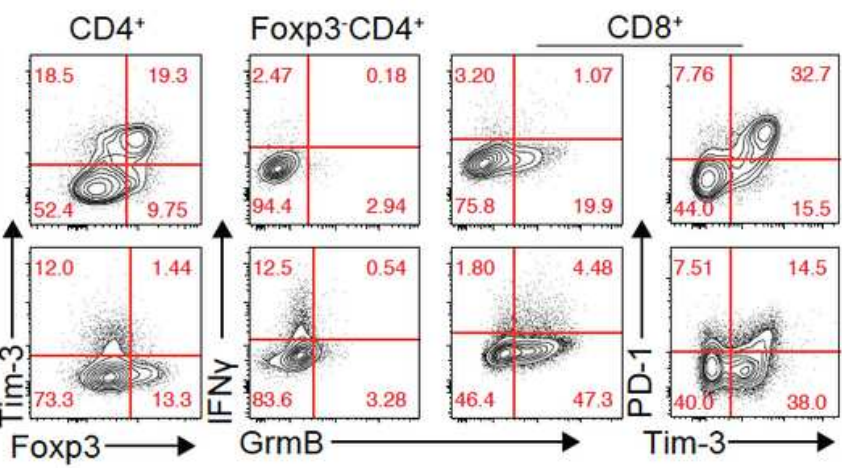

E
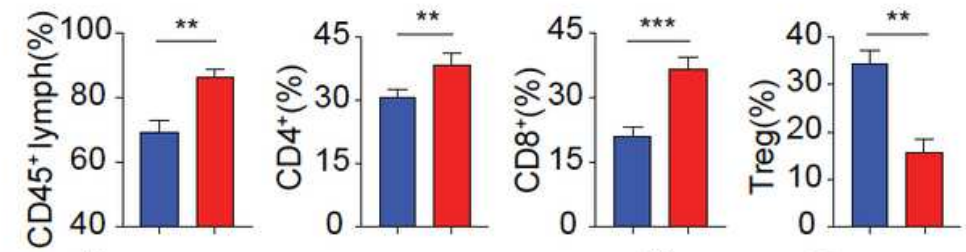

$\mathbf{F}$
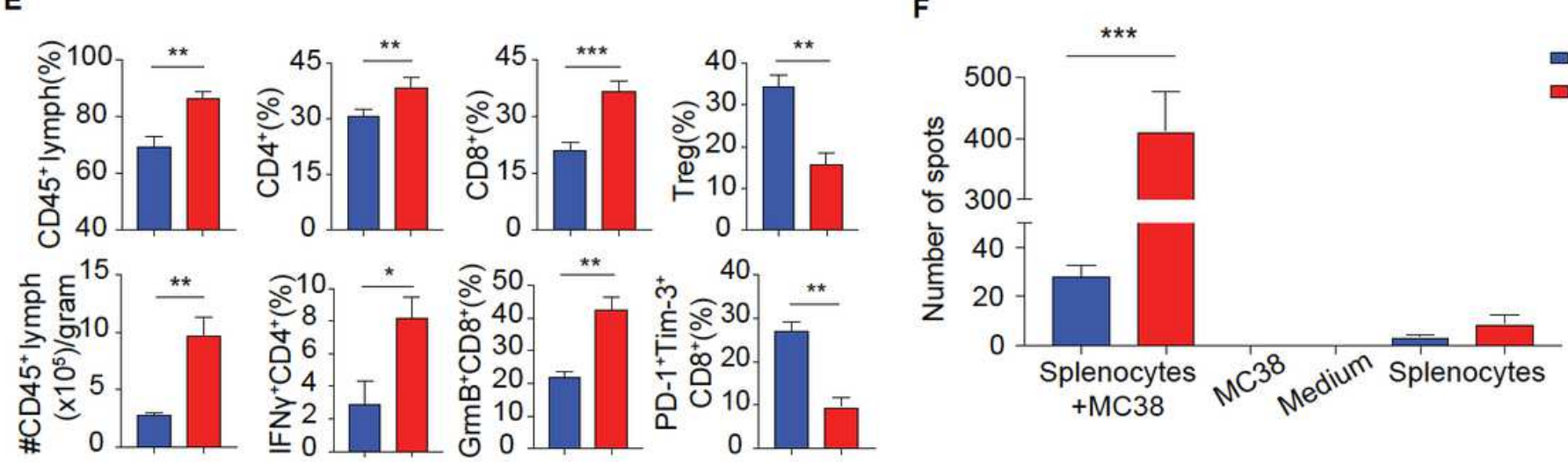

$\square T^{+/-} F^{\text {cre }}$

- $T^{1 /} F^{\text {cre }}$

\section{Figure 3}

(A) Survival curves of Tf/- FCre and T+/+ FCre mice ( $n=20$ and 13, respectively), Log-rank test. (B) Growth curves of MC38 tumors in Tf/- FCre and T+/- FCre mice. Data were presented as mean $\pm S E M, n=5$, Student's t test. (C) Growth curves of MC38 tumors in Tf/f FCreErt2 and FCreErt2 mice. Data were presented as mean \pm SEM, $n=5$, Student's t test. (D-F) MC38 tumors (6 8 mm in diameter) were analyzed by flow cytometry and splenocytes were analyzed by Elispot assay. (D-E) Percentage and total number of tumor infiltrating CD45+ lymphocytes, percentages of CD4+ T, CD8+ T cells and Treg cells and expression of granzyme B, IFN-g, PD-1, and Tim-3 on Foxp3-CD4+ and CD8+ T cells were shown. Data were presented as mean $\pm S E M, n=3-4$. ${ }^{*} P<0.05, * \star P<0.01, * \star * P<0.001$, Student's $t$ test. (F) Numbers of tumor- 
antigen specific IFN-g producers in the splenocytes were obtained by the Elispot assay $(n=3-4)$. Data were presented as mean $\pm S E M, * P<0.05, * \star P<0.01, * \star \star P<0.001$, Student's $t$ test.

A

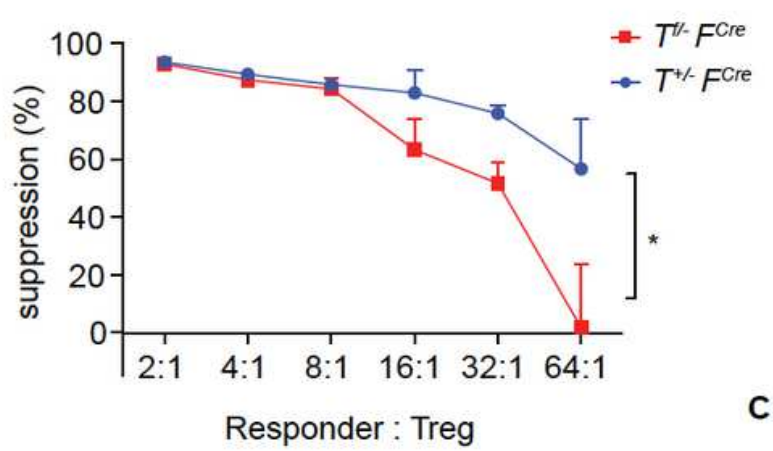

D

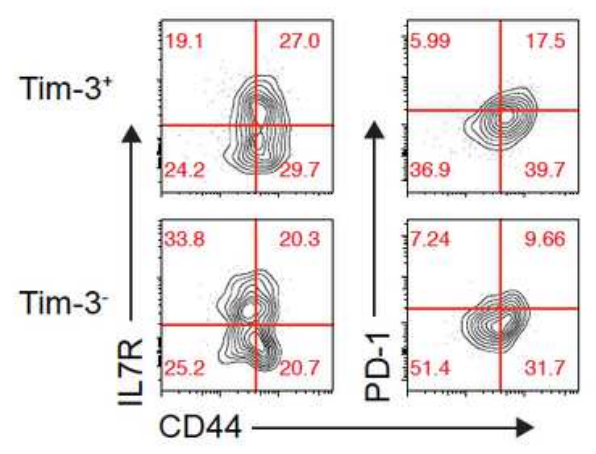

C
B
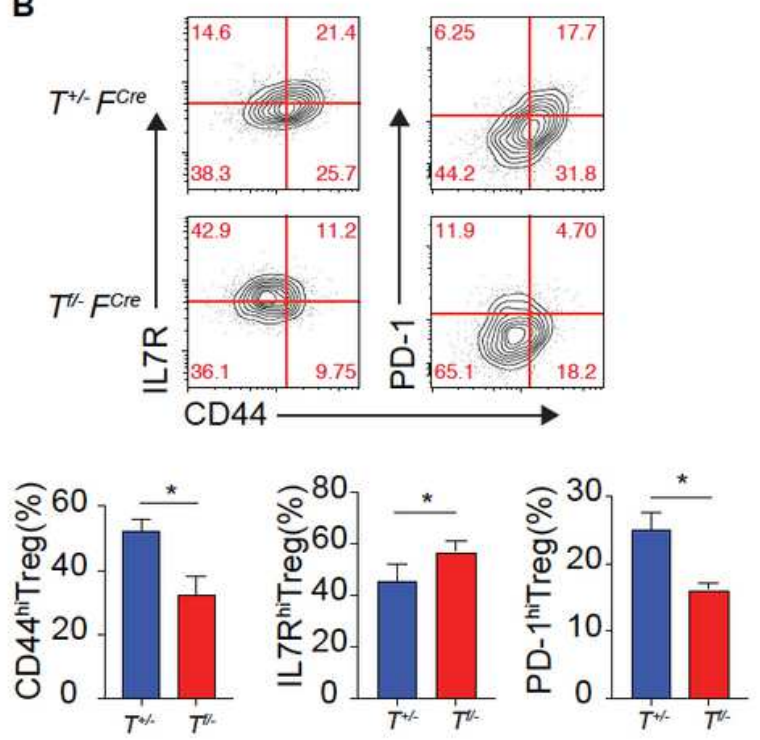

E

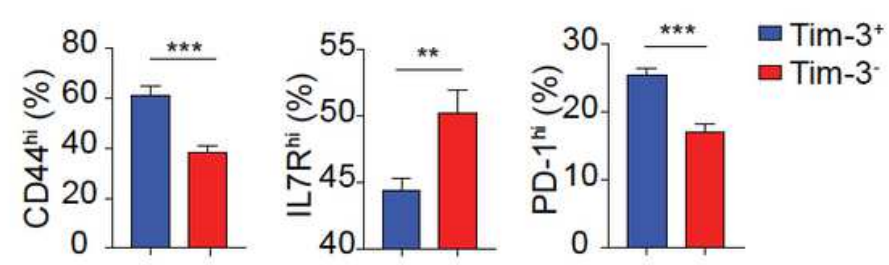

$\mathbf{F}$

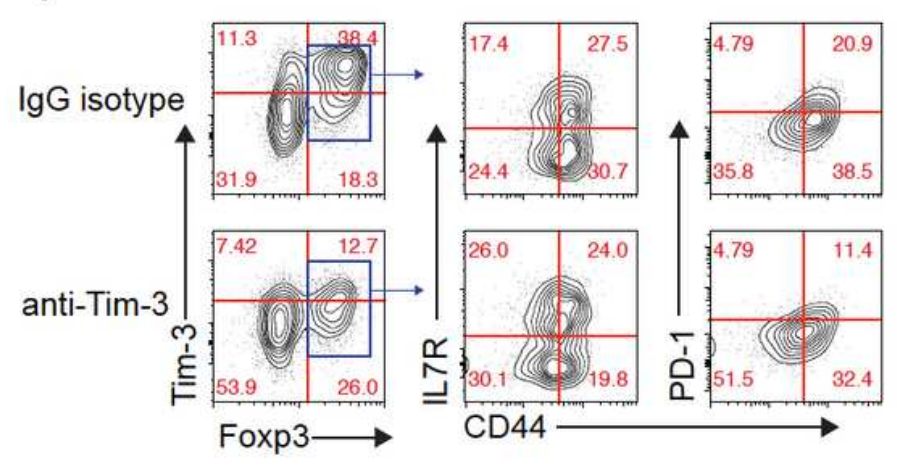

G

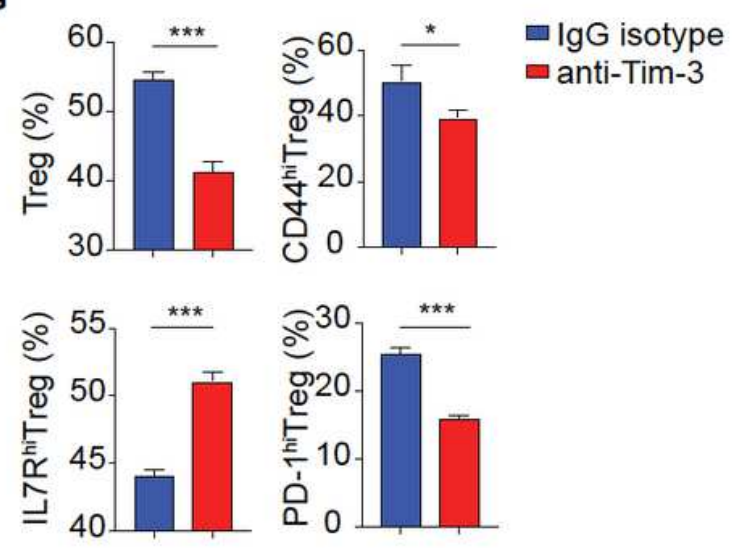

\section{Figure 4}

Blockade of Tim-3 function diminished the number and suppression activity of Treg cells in the TME. (A) Tumoral Treg cells were sorted from Tf/- FCre and T+/- FCre MC38 tumor-bearing mice $(6 \sim 8 \mathrm{~mm}$ in diameter) and assayed in a suppression assay, data were representative of 3 independent experiments with similar results. ${ }^{*} \mathrm{P}<0.05$, Student's $t$ test. (B-C) CD44, IL7R and PD-1 expression on tumoral Treg cells (as in A gated on CD45+CD4+Foxp3+) from Tf/- FCre and T+/- FCre MC38 tumor-bearing mice ( $\mathrm{n}=3-4)$. Data were presented as mean $\pm S E M,{ }^{*}<<0.05$, Student's $t$ test. (D-E) C57BL/ 6 mice were inoculated with 
MC38 tumors ( 8mm in diameter) and Tim-3+ and Tim-3- tumoral Tregs (gated on CD45+CD4+Foxp3+) were analyzed for CD44, IL7R and PD-1 expression by flow cytometry $(n=3-4)$. Data were presented as mean $\pm S E M, * * P<0.01, * * * P<0.001$, Student's $t$ test. (F-G) C57BL/ 6 mice were inoculated with MC38 tumors, when diameter reached about $8 \mathrm{~mm}$, mice were treated with anti-Tim- 3 or IgG control. 24h later, tumoral Tregs cells were analyzed for CD44, IL7R and PD-1 expression $(n=3)$. Data were presented as mean $\pm S E M, * P<0.05, * * * P<0.001$, Student's $t$ test.

A

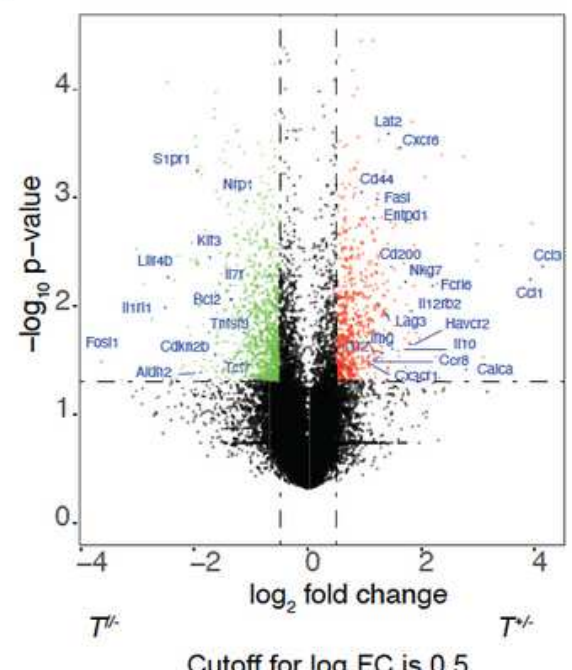

The number of up gene in $T^{+/}$is 955

The number of up gene in $T^{/ /}$is 675

C

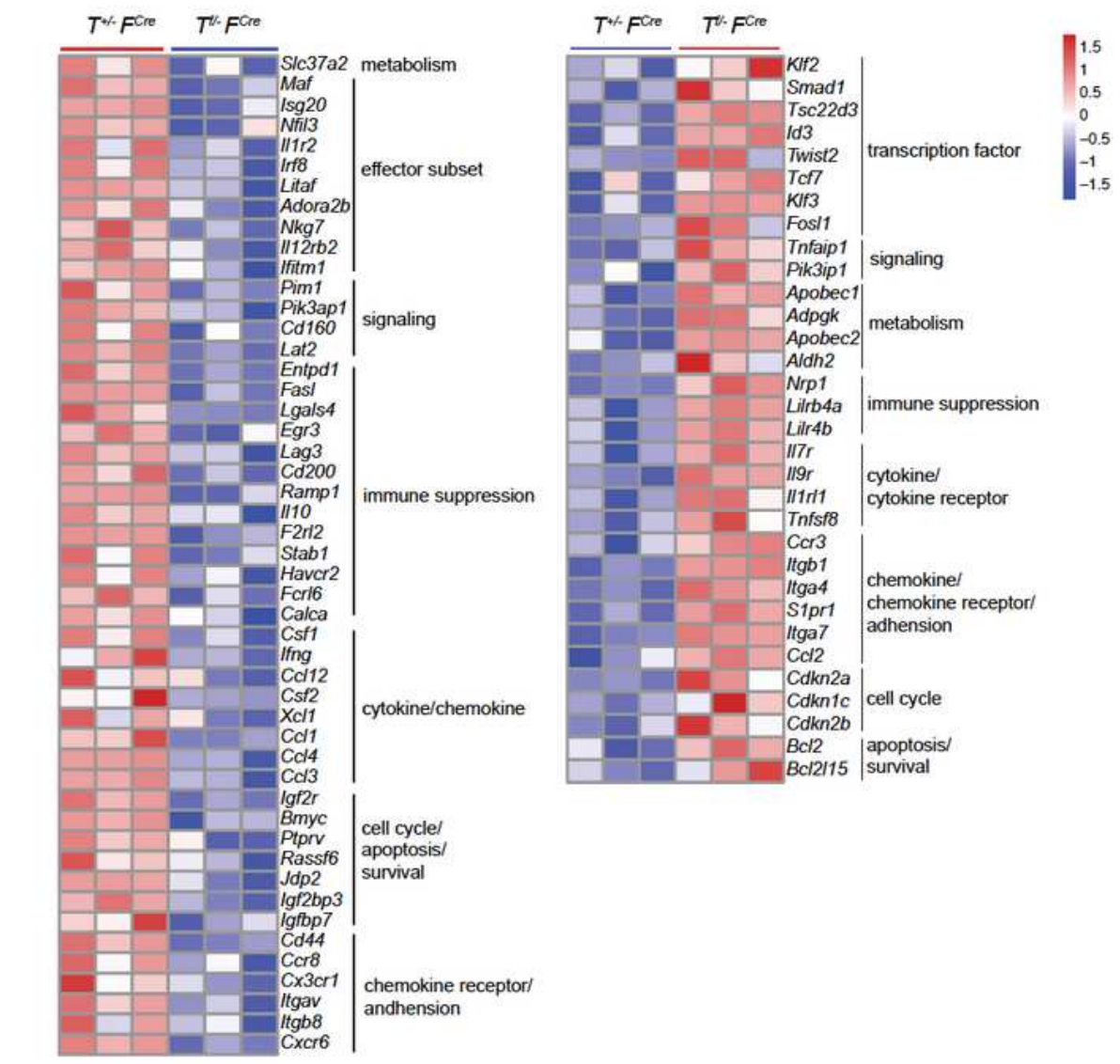

B
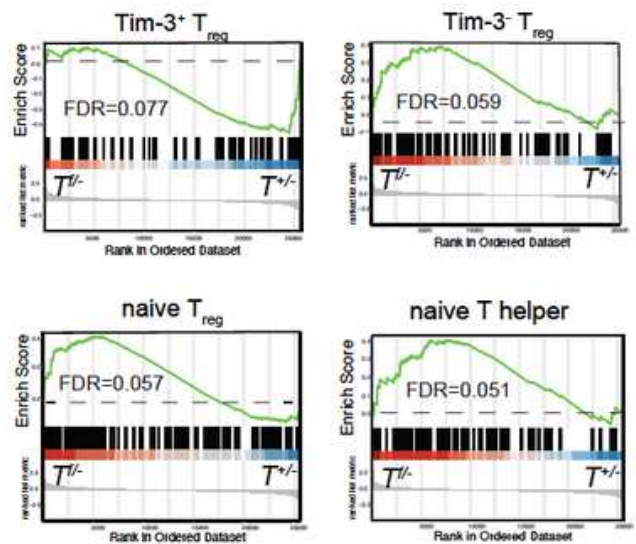

Rank in Ordeond Domase

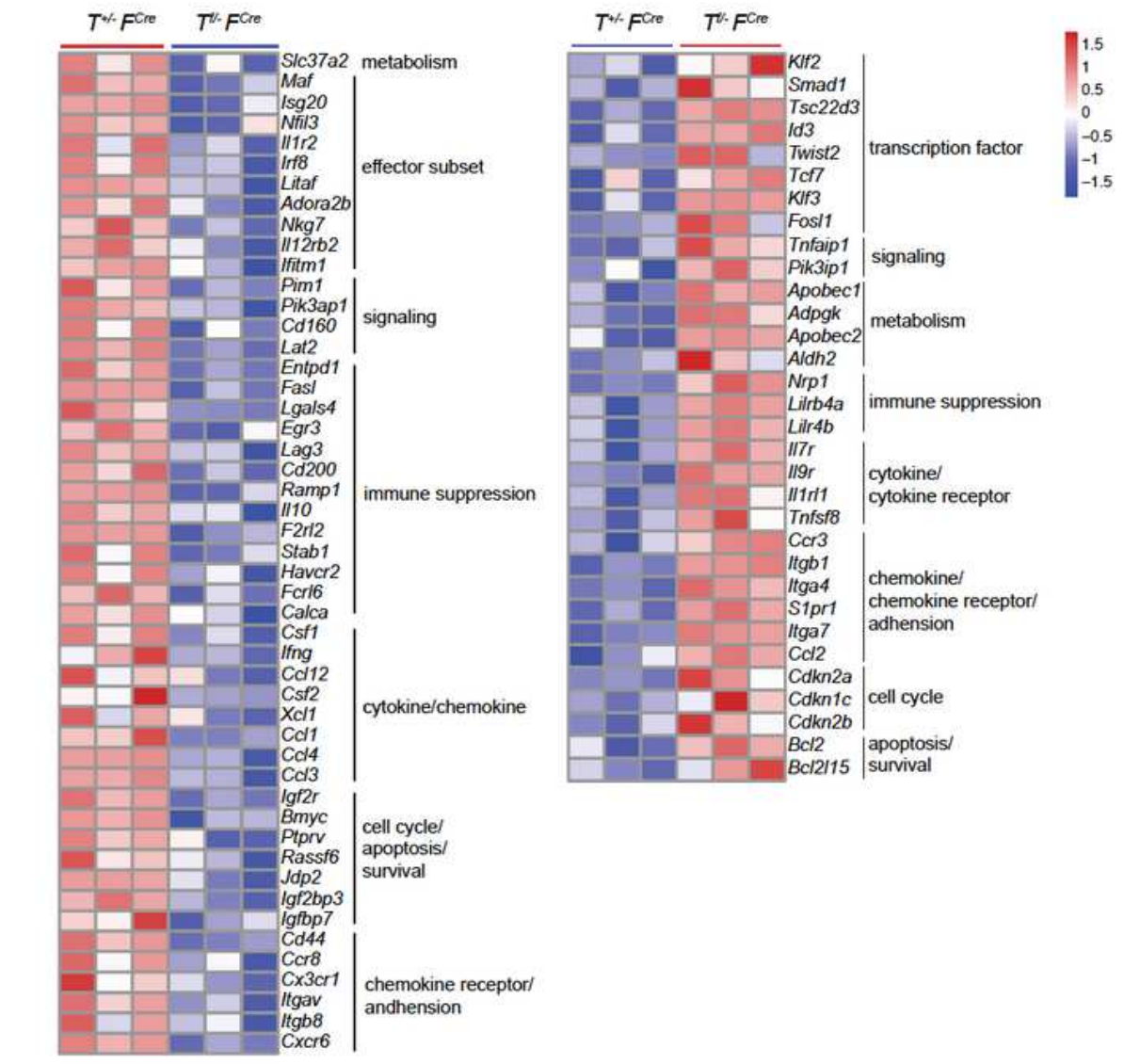


Transcriptome analysis of the Tim-3-driven molecular program in tumoral Treg cells. (A-C) Bulk RNA sequencing analysis of tumoral Tregs from Tf/- FCre and T+/- FCre MC38 tumor-bearing mice $(6 \sim 7 \mathrm{~mm}$ in diameter) $(n=3)$. (A) Volcano plots showing enrichment of DEGs in Tf/- FCre and T+/- FCre Tregs. DEGs with Benjamini-Hochberg adjusted $P$ value $<0.05$ and absolute log2 (fold change) (log2FC) ${ }^{3} 0.5$ were selected for further analysis. (B) GSEA analysis of DEGs enriched in Tf/- FCre and T+/- FCre Tregs with indicated gene signatures. $P$ value? $(C)$ Heatmap showing deferentially expressed genes in $\mathrm{Tf} /-\mathrm{FCre}$ or $\mathrm{T}+/$ - FCre Treg cells. The genes were selected based on functional categories and adjusted $\mathrm{P}$ value $<0.05$ and $\log 2 \mathrm{FC}^{3} 1$.

A

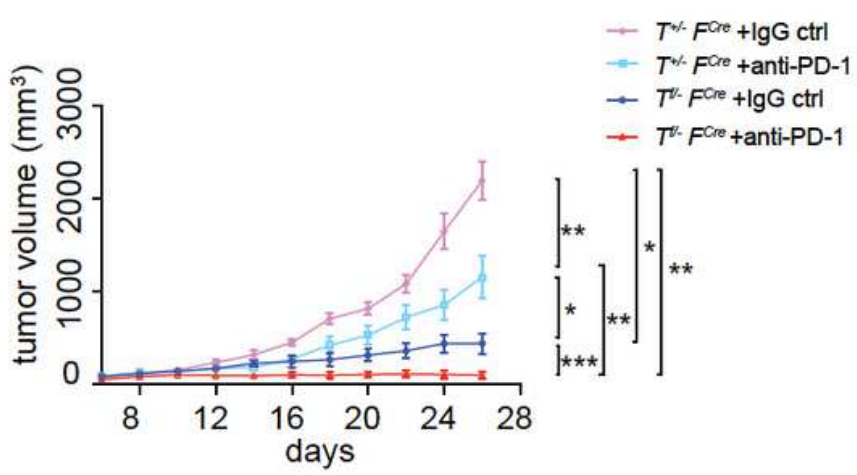

C

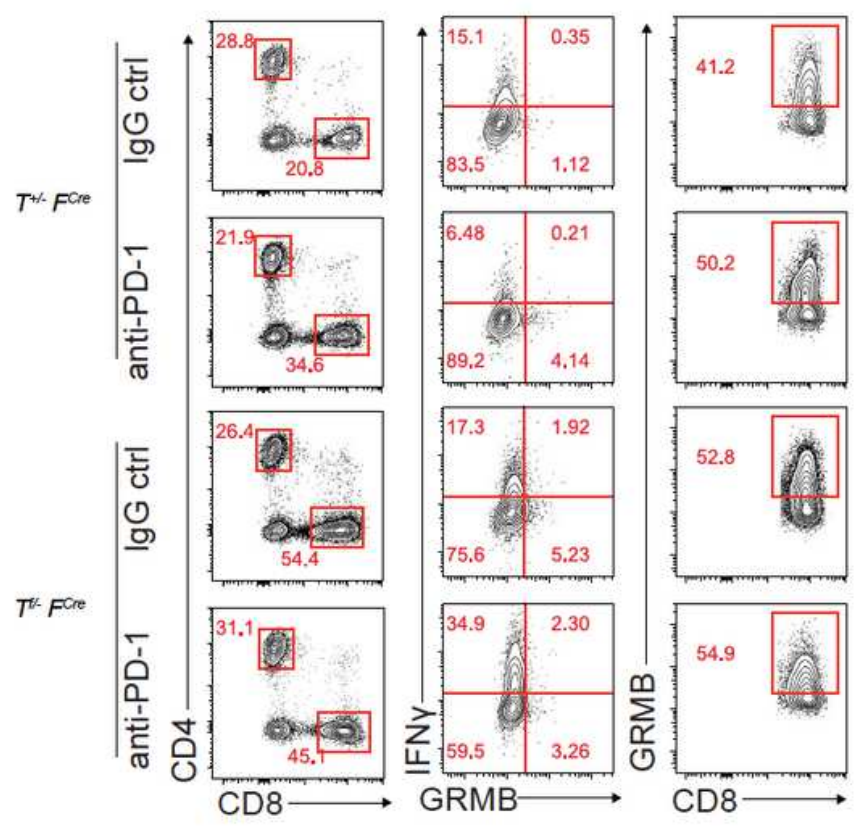

B

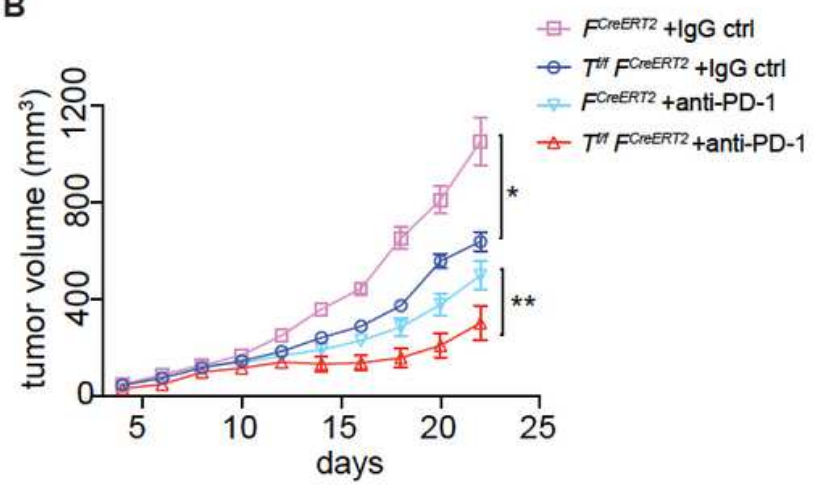

D
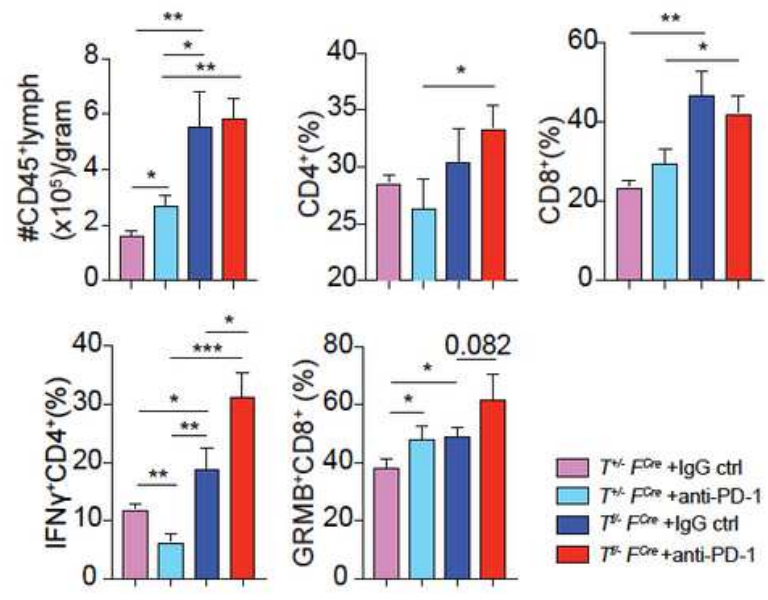

$\square T^{*}$ Fare + lgG ctrl

$\square T^{\text {th }}$ Fre +anti-PD-1

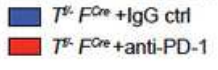

\section{Figure 6}

Tim-3 deletion in Treg cells synergized with PD-1 mAbs in tumor immunotherapy. (A-B). Mean volumes of MC38 tumors treated with IgG isotype or PD-1 mAbs in T+/- FCre and Tf/- FCre mice (A, n=5-7) and 
Foxp3CreERT2 and Tf/f Foxp3CreERT2 mice $(B, n=5-6)$. Data were presented as mean $\pm S E M, * P<0.05$, ** $\mathrm{P}<0.01$, ${ }^{* \star *} \mathrm{P}<0.001$, One-way ANOVA test and Student's $t$ test. (C-D). $T+/-$ FCre and Tf/- FCre mice were inoculated with MC38 tumors, when tumor sizes reached $6 \mathrm{~mm}$, mice were treated with PD-1 mAbs or control IgG for a total of 2 times. 72 hours later, tumors were isolated and analyzed by flow cytometry. Representative flow plots and statistics of CD45+, CD4+ and CD8+ lymphocytes and IFN-g, granzyme B production were showed $(n=4)$. Data were presented as mean $\pm S E M, * P<0.05, \star \star P<0.01, \star \star \star P<0.001$, Student's $t$ test were performed. Data are representative three independent experiments. 